\title{
Effect of Western Diet and Caloric Substitution with Apple Pomace on Fatty Acid Composition and Gene Expression in the Hypothalamus of Growing Female Sprague-Dawley Rats
}

\author{
Ayad Abdulhamid Alawadi \\ West Virginia University, ayalawadi@mix.wvu.edu
}

Follow this and additional works at: https://researchrepository.wvu.edu/etd

Part of the Nervous System Diseases Commons, and the Nutritional and Metabolic Diseases

Commons

\section{Recommended Citation}

Alawadi, Ayad Abdulhamid, "Effect of Western Diet and Caloric Substitution with Apple Pomace on Fatty Acid Composition and Gene Expression in the Hypothalamus of Growing Female Sprague-Dawley Rats" (2020). Graduate Theses, Dissertations, and Problem Reports. 7957.

https://researchrepository.wvu.edu/etd/7957

This Thesis is protected by copyright and/or related rights. It has been brought to you by the The Research Repository @ WVU with permission from the rights-holder(s). You are free to use this Thesis in any way that is permitted by the copyright and related rights legislation that applies to your use. For other uses you must obtain permission from the rights-holder(s) directly, unless additional rights are indicated by a Creative Commons license in the record and/ or on the work itself. This Thesis has been accepted for inclusion in WVU Graduate Theses, Dissertations, and Problem Reports collection by an authorized administrator of The Research Repository @ WVU. For more information, please contact researchrepository@mail.wvu.edu. 


\title{
Effect of Western Diet and Caloric Substitution with Apple Pomace on Fatty Acid Composition and Gene Expression in the Hypothalamus of Growing Female Sprague- Dawley Rats
}

\author{
Ayad A. Alawadi \\ Thesis submitted to the \\ Davis College of Agriculture, Natural Resources, and Design \\ at West Virginia University \\ in partial fulfillment of the requirements for the degree of \\ Master of Science in \\ Nutritional and Food Sciences \\ Janet Tou, $\mathrm{PhD}$, Chair \\ Vagner Benedito, PhD \\ Jacek Jaczynski, PhD \\ R. Chris Skinner, PhD
}

Division of Animal and Nutritional Sciences

Morgantown, WV

2020

Keywords: Western diet, apple pomace, hypothalamus, neurodegenerative diseases, functional foods.

Copyright 2020 Ayad A. Alawadi 


\title{
Abstract \\ Apple Pomace Favorably Alters Brain Fatty Acid Composition and Gene Expression in the Hypothalamus of Growing Female Sprague-Dawley Rats Fed a Western Diet
}

\author{
Ayad A. Alawadi
}

The hypothalamus plays a major role in regulating food-intake and energy-expenditure. Hypothalamic dysfunction implicated in neurodegenerative diseases is characterized by dietinduced neuroinflammation and energy dysregulation. Apple pomace, a waste byproduct of processing, is rich in polyphenols and soluble fibers, has the potential to ameliorate diet-inducing inflammation. The objective of this study was to determine the effects of Western diet and caloric substitution of Western diet with $10 \%$ apple pomace on brain fatty acid composition and gene expression related to hypothalamic function and health in growing female rats. Growing female Sprague-Dawley rats (age 22-29 days) were randomly assigned ( $\mathrm{n}=8$ rats/group) to consume purified AIN-93G (control), Western diet, or Western calorically substituted with 10\% apple pomace (Western/AP) diets for 8 weeks. Brain lipid content and fatty acid profile analysis were measured. Differentially expressed genes were measured in the hypothalamus using RNASeq. Results showed Western/AP diet consisted of the highest amount of soluble fibers and polyphenols. Brain oleic acid was highest in rats fed Western/AP diet $(p<0.0005)$. RNA-seq results comparing the hypothalamus of rats fed Western to Western/AP showed 15 differentially expressed genes, of which 5 genes: phospholipase D family member 5 (PLD5), synuclein alpha (Snca), NADH dehydrogenase (ubiquinone) fe-s protein 6 (Ndufs6), choline O-acetyltransferase (Chat), and frizzled class receptor 6 (Fzd6) were implicated in neurodegenerative diseases. Apple pomace attenuated the upregulation of Snca $(q<0.05)$, Chat $(q<0.05)$, and Ndufs6 $(q<0.05)$ gene expression, as well as downregulated PLD5 $(q<0.06)$, and Fzd6 $(q<0.05)$ gene 
expression. Results suggested apple pomace constitutes reduced neurodegeneration, acetylcholine impairment, and mitochondrial dysfunction through the modulation of brain lipid content and profile. In conclusion, the results provide evidence that caloric substitution of Western diet with apple pomace has the potential to attenuate the pathogenesis of neurodegenerative diseases. Based on pre-clinical evidence, apple pomace has the potential to be a sustainable functional food for brain health. 


\section{Acknowledgments}

Abu Hurairah narrated that the Messenger of Allah said :

"Whoever is not grateful to the people, he is not grateful to Allah."

I am grateful to my Father, Abdulhamid Alawadi, and my Mother, Sarah Alhatem, for their unwavering guidance and support during my study abroad. Your patience and care cannot be overestimated. I am thankful to have supportive sisters and brothers that never let me down.

I am also immensely grateful for Dr. Janet Tou for accepting me as her first international student and for delivering the education, knowledge, and much more that I was seeking for. Especially during the pandemic, your relentless help and emotional support are exceedingly appreciated. Thank you for placing your trust in me.

I would like to thank my committee members, Dr. Vagner Benedito, for the constant help in my RNA experiments and Dr. Jacek Jaczynski, for the delightful discussions about food science. I would like to extend my thanks to Dr. Chris Skinner for his previous work on the experiments and for providing the necessary materials to do my research.

Thanks should also go to Dr. Derek Warren for teaching me how to conduct the experiments for my research. Thanks to Nariman Ktil for her supportive assistance in the lab, and thanks to my close friends who me helped through this journey.

Thank you Kuwait Cultural Office and Ministry of Education for providing the financial support to continue my studies at WVU. I would like to thank WVU for providing such a welcoming and diverse environment that enabled me to meet extraordinary professors and earn my degree. 


\section{Table of Contents}

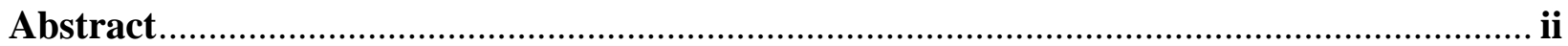

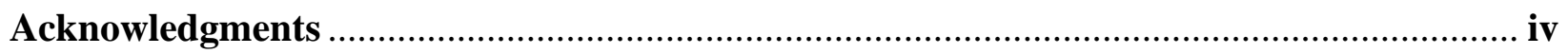

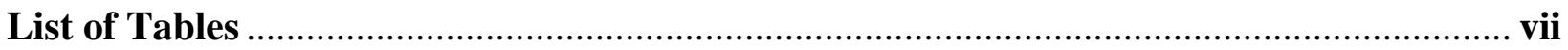

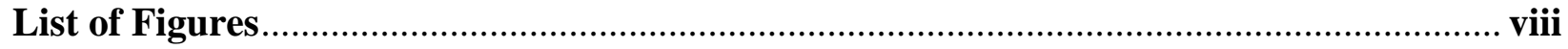

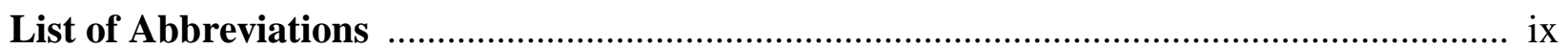

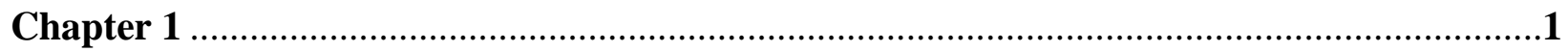

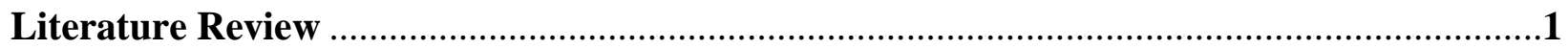

Western Diet Influences Brain Function and Neurodegeneration .......................................1

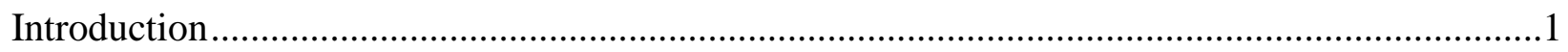

High Fat Diets on Brain Function and Neurodegeneration ......................................................

High Sugar Diets on Brain Function and Neurodegeneration ……...........................................

Dietary Fibers on Brain Function and Neurodegeneration ......................................................11

Polyphenols on Brain Function and Neurodegeneration ..........................................................14

Caloric Substitution of Western Diet with Apple Pomace …………….......................................17

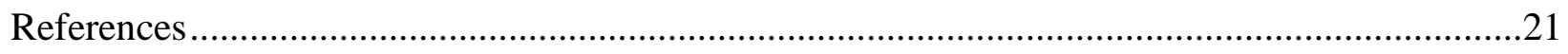

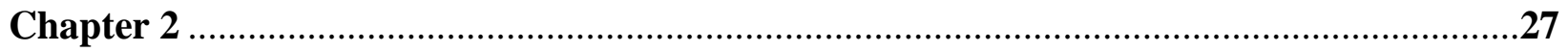

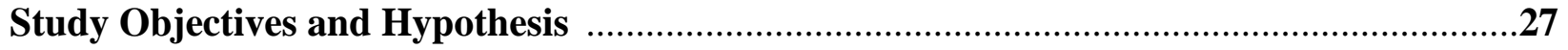

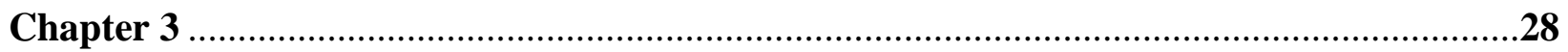

Apple Pomace Favorably Alters Brain Fatty Acid Composition and Gene Expression in the Hypothalamus of Growing Female Sprague-Dawley Rats Fed a Western Diet ...................28

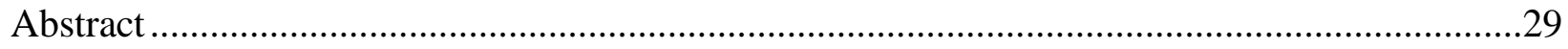

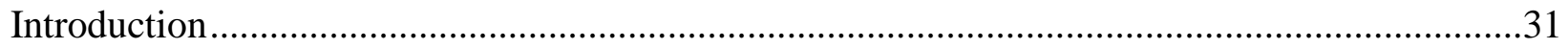

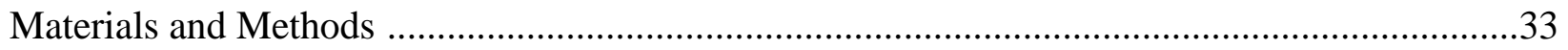

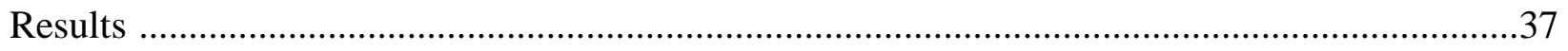




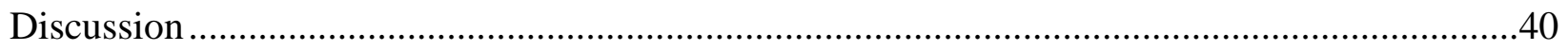

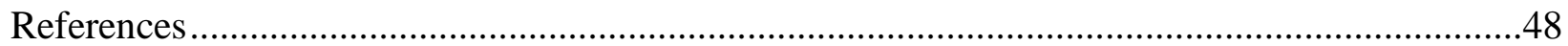

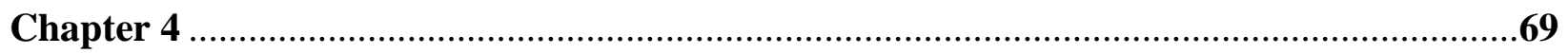

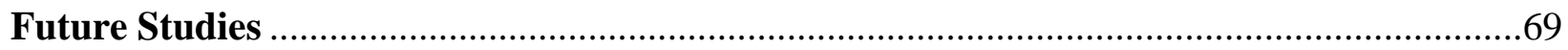




\section{List of Tables}

\section{Chapter 3}

$\begin{array}{ll}\text { Table } 1 . & 54\end{array}$

Table 2. 55

Table 3.

$\begin{array}{ll}\text { Table } 4 . & 57\end{array}$

$\begin{array}{ll}\text { Table } 5 . & 58\end{array}$

Table $6 . \quad 59$ 


\section{List of Figures}

\section{Chapter 3}

Figure 1.

Figure 2.

Figure 3.

Figure 4.

Figure 5.

Figure 6.

Figure 7. 


\section{List of Abbreviations}

\section{Chapter 1}

\begin{tabular}{|c|c|}
\hline $\mathrm{AgRP}$ & Agouti-related protein \\
\hline A-LA & A-linolenic acid \\
\hline AMDR & Acceptable Macronutrient Distribution Range \\
\hline AP & Apple pomace \\
\hline APP & Amyloid protein precursor \\
\hline ARA & Arachidonic acid \\
\hline Chat & Choline O-acetyltransferase \\
\hline CNS & Central nervous system \\
\hline DHA & Docosahexaenoic \\
\hline DNL & De Novo Lipogenesis \\
\hline EPA & Eicosapentaenoic acid \\
\hline FOXO1 & Forkhead box protein $\mathrm{O} 1$ \\
\hline HCD & High carbohydrate diet \\
\hline HFD & High fat diet \\
\hline HFRD & High fructose diet \\
\hline HSD & High sucrose diet \\
\hline Iba1 & Ionized calcium binding adaptor molecule \\
\hline ICV & Intracerebroventricular \\
\hline $\mathrm{IL}$ & Interleukin \\
\hline LA & Linoleic acid \\
\hline MUFA & Monounsaturated fatty acids \\
\hline NAFLD & Non-alcoholic fatty liver disease \\
\hline NASH & Non-alcoholic steatohepatitis \\
\hline POMC & Pro-opiomelanocortin \\
\hline PS1 & Presenilin 1 \\
\hline PUFA & Polyunsaturated fatty acids \\
\hline RDA & Recommended Dietary Allowance \\
\hline ROS & Reactive oxygen species \\
\hline SCD1 & Stearoyl-CoA desaturase- 1 \\
\hline SCFA & Short-chain fatty acids \\
\hline SFA & Saturated fatty acids \\
\hline STAT3 & Signal transducer and activator of transcription 3 \\
\hline TLR & Toll-like receptor \\
\hline $\mathrm{TNF}-\alpha$ & Tumor necrosis factor- $\alpha$ \\
\hline VLDL & Very low-density lipoproteins \\
\hline
\end{tabular}




\section{Chapter 3}

\begin{tabular}{|c|c|}
\hline AgRP & Agouti-related protein \\
\hline AIN & American Institute of Nutrition-93G diet \\
\hline $\mathrm{AP}$ & Apple pomace \\
\hline ARA & Arachidonic acid \\
\hline Cart & Cocaine-and amphetamine-regulated transcript \\
\hline Chat & Choline O-acetyltransferase \\
\hline CNS & Central nervous system \\
\hline $\mathrm{ddH}_{2} \mathrm{O}$ & Deionized water \\
\hline DHA & Docosahexaenoic \\
\hline DNL & De Novo Lipogenesis \\
\hline EPA & Eicosapentaenoic acid \\
\hline Faim & Fas apoptotic inhibitory molecule \\
\hline FOXO1 & Forkhead box protein $\mathrm{O} 1$ \\
\hline Fzd6 & Fizzled class receptor 6 \\
\hline GLC & Gas liquid chromatography \\
\hline $\mathrm{HCA}$ & Hierarchical clustering analysis \\
\hline InsR & Insulin receptors \\
\hline Jak3 & Janus kinase 3 \\
\hline LepR & Leptin receptors \\
\hline $\mathrm{Mc} 4 \mathrm{r}$ & Melanocortin 4 receptor \\
\hline MUFA & Monounsaturated fatty acids \\
\hline NAFLD & Non-alcoholic fatty liver disease \\
\hline Ndufs6 & NADH dehydrogenase (ubiquinone) fe-s protein 6 \\
\hline Npy & Neuropeptide Y \\
\hline PCA & Principal component analysis \\
\hline Pi3k & Phosphoinositide 3-kinases \\
\hline PLD5 & Phospholipase D family member 5 \\
\hline POMC & Pro-opiomelanocortin \\
\hline PUFA & Polyunsaturated fatty acids \\
\hline ROS & Reactive oxygen species \\
\hline SFA & Saturated fatty acids \\
\hline Snca & Synuclein alpha \\
\hline STAT3 & Signal transducer and activator of transcription 3 \\
\hline
\end{tabular}




\section{Chapter 1}

\section{Literature Review}

\section{Introduction}

The brain regulates various activities in the body including energy homeostasis and cognitive functions [1]. In order to maintain these activities; the brain is a high-energy organ requiring $20 \%$ of the body's total energy expenditure [1]. Energy sources are metabolized in the central nervous system (CNS) by neurons and glial cells. In the fed state, the major energy sources for the brain are glucose and lactate while ketone bodies are the major energy substrate during the fasted state [2]. These energy sources are preferred by neuronal mitochondria because their rapid and efficient catabolism meets the high demands of neuronal processes, such as action potential and neurotransmitters recycling $[2,3]$. Despite the high energy demands of the brain, fatty acid oxidation is not preferred by the CNS due to: (1) use in neuronal and glial cells as bilayer membranes and myelin sheaths to maintain homeostatic functions, (2) maintenance of neuronal cells rather than energy metabolism, and (3) slower fatty acid oxidation by the mitochondria compared to glucose, lactate, and ketone body oxidation due to enzymatic requirements for transporting fatty acids into the mitochondrial complex as well as high oxygen demand to produce adenosine triphosphate $[1,3]$.

The hypothalamus plays a major role in regulating energy sources because it sustains energy requirements for the CNS as well as for the body $[4,5]$. Energy regulation by the hypothalamus is controlled peripherally through extra-neuronal tissues by insulin signals from the pancreas and by leptin signals from the adipose [6]. Both leptin and insulin signals are induced through diet and in turn, function to control food intake and energy expenditure [6]. In 
the hypothalamus, the arcuate nucleus contains Pro-opiomelanocortin (POMC) and Agoutirelated protein neurons $(\mathrm{AgRP})$ regulated by leptin and insulin by binding to their respective receptors [7]. In AgRP neurons, lack of binding of leptin and insulin to their receptors activates Forkhead box protein O1 (FOXO1) which binds to POMC promoter to induce appetite [7]. In POMC neurons, binding of leptin to leptin receptors activates signal transducer and activator of transcription 3 (STAT3) and phosphoinositide 3-kinases pathways [7]. This leads to the inactivation of FOXO1 which induces satiety [7]. Upregulation of POMC neurons induces anorexigenic effects by inhibiting AgRP neurons [7]. Also, stimulation of POMC neurons releases $\alpha$-melanocyte stimulating hormone, which inhibits food intake and promotes weight loss by increasing energy expenditure [8]. Disruption, such as inflammation, in hypothalamic neurons controlling satiety and appetite, leads to imbalances in energy homeostasis as well as the development of metabolic syndromes [5]. Therefore, specific dietary macronutrients can affect brain energy metabolism, function, and homeostasis.

The Western diet is a calorically dense diet. Although there is no standard definition of the Western diet, it is characterized by processed foods that are high in fat (e.g. animal-derived saturated fatty acids) and high in refined sugars (e.g. sucrose and fructose), but low in protein, dietary fibers, micronutrients, and antioxidants [4]. By providing excessive energy from fat or refined sugar, the Western diet induces mitochondrial dysfunction and neuroinflammation in the hypothalamus, which has been suggested to contribute to neurodegenerative diseases [1, 3-5]. Studies have reported patients with metabolic syndromes, such as obesity and type 2 diabetes mellitus, are at higher risk of developing neurodegenerative diseases due to their proinflammatory state [9]. 
Neurodegenerative diseases are incurable debilitating chronic conditions caused by neuronal inflammation leading to cell death in the CNS [1]. Neurodegenerative diseases are characterized by accumulation and aggregation of misfolded proteins forming Lewy bodies in neuronal cells via the amyloidogenic pathway [10]. Cellular inflammation upregulates $\beta$ secretase 1 and activates $\gamma$-secretase gene expression, a component of presenilin, in the amyloidogenic pathway producing $\beta$-amyloid and other misfolded proteins in neuronal cells [10]. Hypothalamic dysfunction and cognitive impairments have often been reported prior to the development of Alzheimer's disease [1, 5, 9]. Chronic low-grade cellular inflammation has been suggested to initiate a cycle of energy dysregulation in the hypothalamus that induces aggregation of misfolded proteins, leading to the onset of neurodegenerative diseases at a later age [9]. Current medications can extend the life expectancy of patients; however, quality of life is not improved [10]. Studies suggest diets high in dietary fiber and antioxidant polyphenols may protect against neuroinflammation leading to hypothalamic dysfunction and neurodegenerative diseases [4]. The aim of this literature review is to assess the effects of saturated fats, refined carbohydrates, (e.g. sucrose and fructose), soluble fibers, and antioxidant polyphenols on hypothalamic function and neurodegenerative diseases.

\section{High Fat Diets Effects on Brain Function and Neurodegeneration}

High-fat diet (HFD) are diets that exceed the Acceptable Macronutrient Distribution Range (AMDR) for adults of 20-35\% daily total kcals [4,11]. HFD consists mainly of long-chain saturated fatty acids (SFAs), palmitic acid (16:0) and stearic acid (18:0), as well as omega-6 polyunsaturated fatty acids (PUFAs), linoleic acid (LA, 18:2n-6) and arachidonic acid (ARA, 20:4n-6). HFD are low in omega-3 PUFAs, $\alpha$-linolenic acid (A-LA, 18:3n-3), eicosapentaenoic 
acid (EPA, 20:5n-3), and docosahexaenoic (DHA, 22:6n-3) [12, 13]. In lipid metabolism, chylomicrons transport dietary triacylglycerols while very-low-density lipoproteins (VLDL) deliver hepatic de novo synthesized triacylglycerols to extrahepatic tissues including the brain [14]. Since the fatty acid composition of chylomicrons and VLDL depends on the type of diet consumed, this suggests a direct effect of diet on brain lipid profile and in turn, brain function $[14,15]$. In the brain, microglia act as immune cells modulating inflammation [10]. Studies showed HFD consumption, particularly long-chain SFAs ( $\geq 16 \mathrm{C})$, activated microglial cells to induce neuroinflammation in the CNS $[5,16]$. In vitro, primary murine microglial cells were treated for $24 \mathrm{~h}$ with SFAs, unsaturated fatty acids, or bovine serum albumin as a negative control. Lipopolysaccharides were used as a positive control to activate microglia and inflammatory markers [16]. Results showed palmitic (16:0) and stearic (18:0) acid induced significantly higher activation of microglia and protein expression of pro-inflammatory cytokines, tumor necrosis factor- $\alpha$ (TNF- $\alpha$ ), and interleukin-6 (IL-6), compared to oleic acid (18:1) and control [16].

Similar results were found in vivo. Mice fed HFD ( $42 \%$ total kcals) consisting of longchain SFAs, palmitic acid (16:0) and stearic acid (18:0), for 16 weeks, showed accumulation of microglial cells in the hypothalamus accompanied by protein expression of TNF- $\alpha$ compared to mice fed a standard chow diet [16]. To determine whether these results were influenced by dietary fats independent of excess caloric intake or weight gain, mice were orally gavaged with radioactive labeled palmitic acid (16:0). Radioactive labeled palmitic acid (16:0) was detected in various tissues including the hypothalamus suggesting dietary lipids accumulation in the brain. This was further confirmed by injecting mice with VLDL containing SFAs labeled with a fluorescent lipophilic dye. Results showed uptake of SFAs by microglial cells in the 
hypothalamus within one hour. The collective study results indicated HFD consumption consisting mainly of long-chain SFAs, palmitic acid (16:0), and stearic acid (18:0), activated microglial cells, and induced production of pro-inflammatory cytokines in the hypothalamus through circulating lipoproteins independent of weight gain in mice [16].

Another in vivo study showed HFD consumption resulted in a faster rate of lipid accumulation and greater neuroinflammation in the brain compared to a high carbohydrate diet (HCD) [17]. Silva-Santi et al. [17] reported mice fed HFD (fat 58\%, carbohydrate 27\% total kcals) or HCD (carbohydrates 76\%, 9\% fat total kcals) for 7, 14, 28, or 56 days showed significantly higher palmitic acid (16:0) and stearic acid (18:0) at day 14 and 28 compared to mice fed HCD. Furthermore, on day 14 and 28 mice fed HFD accumulated significantly higher brain lipid content (76\%) compared to mice fed HCD on day 14 (13\%) and 28 (64\%). However, at day 56, palmitic, stearic acid, and brain lipid content were similar between mice fed HFD and HCD. Results suggested that long-term consumption of both HFD and HCD produced no significant differences in brain SFA content. The study also measured inflammatory marker index, a summation of each pro-inflammatory marker gene expression (e.g. TNF- $\alpha$ and IL-6) divided by an anti-inflammatory marker (IL-10). Results showed a $46 \%$ significantly higher inflammatory marker index at day 56 in mice fed HFD compared to HCD, indicating HFD consumption induced higher neuroinflammation than HCD [17].

Long-chain SFAs and neuroinflammation have also been implicated in the disruption of energy homeostasis in the hypothalamus $[5,13]$. The hypothalamus regulates food intake and energy expenditure by receiving peripheral signals (e.g. leptin) to maintain energy balance by inducing anorexigenic or orexigenic effect [5]. To investigate whether SFAs disrupts energy 
regulation in the hypothalamus, mice (aged 10 weeks) maintained on a standard chow diet were given an intracerebroventricular (ICV) injection of palmitic acid (25 pmol) and compared to control mice given an ICV injection of saline [18]. Leptin signaling and inflammatory response were measured $24 \mathrm{~h}$ after ICV administration. Results found central leptin resistance indicated by decreased protein expression of downstream mediators in food intake pathway, STAT3 and FOXO1, and increased levels of protein expression of inflammatory cytokine, TNF- $\alpha$, in the hypothalamus. The results suggested long-chain SFAs disrupted energy homeostasis in the hypothalamus due to diet-induced leptin resistance. In addition, the study investigated the effects of palmitic acid (16:0) induced leptin resistance in the brain on liver functions. Pre-ICV administration of palmitic acid (16:0) also decreased hepatic gene expression of gluconeogenesis enzymes: glucose 6-phosphatase and phosphoenolpyruvate carboxykinase, glucose transporter 2, and de novo lipogenesis (DNL) enzymes, fatty acid synthase, and stearoyl-CoA desaturase-1 (SCD1) [18]. Collectively, the study results showed palmitic acid (16:0) induced neuroinflammation in the hypothalamus resulted in energy dysregulation in the CNS and liver.

In addition to hypothalamus dysfunction, HFD has been implicated in the development of neurodegenerative diseases $[5,19]$. Transgenic mice expressing human amyloid protein precursor (APP) and presenilin 1 (PS1) were fed HFD (54\% total kcals) or control (10\% total kcals) for 10 weeks. Transgenic mice fed HFD showed brain oxidative stress-related to mitochondrial dysfunction and increased deposition of $\beta$-amyloid proteins compared to the control [19]. Similar findings were reported for non-transgenic C57BL/6J mice consuming HFD (45\% total kcals) for 65 weeks. Results showed significantly increased $\beta$-amyloid deposition in the brain mimicked the onset development of Alzheimer's disease in mice fed HFD compared to mice fed a control diet (18\% total kcals) [20]. 
Other hallmarks ascribed to Alzheimer's disease is the decrease in the synthesis of the neurotransmitter acetylcholine in the cholinergic neurons [21]. Cholinergic neurons are innervated throughout the CNS, which are implicated in many brain activities including cognitive processes and energy regulation as well as the synthesis of acetylcholine [21, 22]. In murine cholinergic neuronal cells, $\beta$-amyloid proteins reduced acetylcholine concentration, and this was accompanied by a decrease in choline acetyltransferase activity (Chat), an enzyme involved in the synthesis of acetylcholine [23]. In contrast, acetylcholinesterase activity, an enzyme responsible for catalyzing acetylcholine to choline and acetate, was unaltered suggesting a reduction in the availability of acetylcholine for neurotransmission which implicates neurodegeneration. However, in vivo, acetylcholinesterase activity was decreased in the hypothalamus of rats fed HFD ( $50 \%$ of fat; $18.5 \%$ carbohydrates by weight) or HCD (44\% of refined-sugar; $4.5 \%$ of fat by weight) for 6 months compared to rats fed a control diet (60\% of carbohydrates; $4.5 \%$ of fat by weight) [24]. Overall, results suggest diet-induced cholinergic dysfunction in the hypothalamus reduces acetylcholine synthesis possibly through the $\beta$-amyloid formation.

Several studies reported the role of hypothalamic dysfunction in the pathogenesis of Alzheimer's disease. Patients with Alzheimer's disease had $\beta$-amyloid aggregation in the brain post-mortem and increased leptin in cerebrospinal fluid. Further, leptin receptors were downregulated compared to similarly healthy-aged controls, suggesting leptin resistance [25]. Diet may play a role in neurodegenerative disease. Undernourished Alzheimer's disease patients, in terms of weight loss, showed deposition of misfolded protein in the hypothalamus [26]. 
Collectively, the reviewed preclinical and human studies suggested that the consumption of HFD directly promotes neuroinflammation and hypothalamic dysfunction leading to neurodegenerative diseases. However, Western diets are also high in sugar, and in turn, HCD has also been suggested to influence brain function and neurodegenerative disease [27].

\section{High Sugar Diets Effects on Brain Function and Neurodegeneration}

The Western diet is high in refined carbohydrates. HCD is defined as carbohydrates consisting of $>65 \%$ of total kcals compared to the AMDR of $45-65 \%$ of daily total caloric intake as carbohydrates of which $10 \%$ is from sugar $[4,11]$. HCD consists mainly of sucrose and fructose with low amounts of complex carbohydrates that include soluble fibers [4]. Glucose is preferred by astrocytes in the CNS as the main source of energy metabolism for maintaining the homeostatic function of neurons [1]. However, when provided in excess, glucose stimulates insulin secretion which promotes DNL in the liver and adipose tissue [28]. The principal pathway regulating DNL in the liver is activated by lipogenic genes involved in carbohydrates metabolism and lipogenesis. Transcriptional factors, sterol regulatory element-binding transcription factor 1 is stimulated by glucose, and carbohydrate-response element-binding protein is stimulated by insulin. The end products of DNL in the liver and adipose tissues are the long-chain SFAs, palmitic acid (16:0). In turn, palmitic acid has been implicated in peripheral and neuroinflammation [28].

Several studies have reported diet-induced chronic liver failure and peripheral inflammation contributes to neuroinflammation [29, 30]. Silva-Santi et al. [31] investigated the effects of mice fed HCD (76\% carbohydrates, $9 \%$ fat total kcals) or HFD (58\% fat, $27 \%$ carbohydrate total kcals) for 7, 14, 28, or 56 days on liver metabolism. Gene expression of 
acetyl-CoA carboxylase 1, the estimated enzyme activity of SCD1, D-6 desaturase, and elongase based on individual fatty acid product/precursor ratios and inflammatory index were assessed. Results showed a significant increase in DNL indicated by downregulation of acetyl-CoA carboxylase 1 gene expression and reduced estimated enzyme activity by precursor/product (SCD1, D-6 desaturase, elongase) in the liver of mice fed HCD compared to HFD. Inflammatory marker index was $71 \%$ significantly higher in the liver of mice fed HCD compared to mice fed HFD, suggesting HCD promotes inflammatory status in the liver [31]. In a follow-up study, Silva-Santi et al. [17] on brain tissue, mice fed HFD showed increased neuroinflammation in the brain compared to $\mathrm{HCD}$; whereas HCD induced peripheral inflammation in the liver, suggesting a link between peripheral inflammation and neuroinflammation in the brain.

To investigate whether peripheral inflammation activates microglial cells as an indicator of neuroinflammation, mice were fed HCD (74.2\% total kcals) for 12 weeks. Results showed microglial cell activation in the brain accompanied by a significant increase in brain TNF- $\alpha$ and IL-6 compared to mice fed a control diet (65\% total kcals) [27]. Further, adiposity-index was significantly higher at day 3 and 12 weeks in mice fed HCD compared to the control diet, suggesting adiposity induced neuroinflammation [27]. In a human cross-sectional study comprising of 117 obese (body mass index $\geq 30$ ) and 83 non-obese (body mass index $<24$ ) individuals. Participants were assessed for a range of inflammatory cytokines. Obese individuals showed significantly increased serum IL-5, IL-10, IL-12, IL-13, Interferon-gamma, and TNF- $\alpha$ compared to non-obese individuals [32]. Both the human and animal studies reviewed suggest chronic consumption of HCD promotes inflammation due to increased adiposity and this, in turn, activates microglial cells inducing neuroinflammation. 
High sucrose diet (HSD) consumption has also been implicated in hypothalamic dysfunction and the development of neurodegenerative diseases. In an animal study, transgenic (APP/PS1) and non-transgenic mice fed HSD (35\% sucrose by weight) for 28 weeks showed a significant adipose gain compared to mice fed the control diet (1.16\% sucrose by weight) [33]. Additionally, serum IL-6 and interleukin 1 beta (IL-1 $\beta$ ) content in the hypothalamus were significantly higher in transgenic mice fed HSD compared to non-transgenic mice fed HSD diet. The results showed mice expressing (APP/PS1) were more susceptible to neuroinflammation induced by HSD intake [33]. Further, microglial cell activation only occurred in transgenic mice fed HSD indicated by significantly upregulation gene expression of ionized calcium-binding adaptor molecule 1 (Iba1). Consequently, POMC gene expression was downregulated which was accompanied by reduced phosphorylated STAT3 suggesting hypothalamic dysfunction due to leptin resistance. Additionally, cortical and serum levels of $\beta$-amyloid were significantly higher in transgenic mice fed HSD compared to the control diet suggesting development of neurodegeneration. Overall, results suggested long-term consumption of HSD increases adiposity which contributes to the cascade of neuroinflammation, hypothalamic dysfunction, and increased levels of $\beta$-amyloid involved in the development of neurodegenerative diseases.

Long-term consumption of fructose has also been implicated in the early development of neuroinflammation and neurodegenerative diseases due to its lipogenic properties independent of excess caloric intake [34]. Female rats were fed high fructose diet (HFRD; 30\% total kcals) compared to $0 \%$ fructose (control) for 12 weeks then mated [35]. Pregnant dams were maintained on their assigned diets. Lactating rat dams were fed a standard chow diet until their offspring were weaned. Rat pups exposed to HFRD in utero showed significantly higher brain gliosis, neuronal cell death, and TNF- $\alpha$ compared to pups exposed to no fructose [35]. Results 
showed in utero HFRD exposure influences offspring brain neuroplasticity and neuroinflammation. Similar effects were found in weanling rats fed HFRD (55\% total kcals) for 10 weeks [36]. Feeding HFRD resulted in significantly higher adiposity, upregulated gene expression of the pro-inflammatory marker (complement component 4B) in the hypothalamus, and increased serum TNF- $\alpha$ compared to rats fed the control diet (0.30\% total kcals) [36]. Results showed growing rats fed HFRD consumption promoted adiposity resulting in peripherally induced neuroinflammation.

Collectivity, pre-clinical studies showed consumption of HSD and HFRD similar to the consumption of the Western diet (e.g. high in sugar as well as fat) increasing adiposity that produced peripheral inflammation, and this, in turn, induced neuroinflammation, and development of neurodegenerative diseases. Another characteristic of the Western diet that may potentially promote neuroinflammation and neurodegenerative diseases is the low dietary fiber content.

\section{Dietary Soluble Fibers on Brain Function and Neurodegeneration}

The recommended dietary allowance (RDA) for dietary fibers is $25-38 \mathrm{~g} /$ day for adults [11]. Fiber intake in the United States population is below the RDA for adults (18 g/day) [37]. The low dietary fiber content of the Western diet has been suggested to contribute to neuroinflammation leading to the onset development of neurodegenerative diseases [4]. In contrast, diets that are rich sources of soluble fibers (e.g. fruits and vegetables) have been reported to decrease the risk of developing neurodegenerative diseases $[4,38]$. The consumption of soluble fibers lowers the absorption of dietary long-chain SFAs as well as bile acids by promoting binding and excretion in the feces [39]. 
One of the major phospholipids in bile acids is phosphatidylcholine which accounts for 95\% phospholipid in humans and $100 \%$ in rats [40]. Phosphatidylcholine plays a key role in providing choline for the synthesis of acetylcholine in the CNS reducing the risk of neurodegeneration [41]. Dietary soluble fiber has been reported to selectively retain phospholipids in the liver while excreting bile acids in the feces to sustain sufficient phosphatidylcholine levels for de novo synthesis of acetylcholine in extra-hepatic tissues, such as the brain $[42,43]$. Rats fed pectin supplemented diet ( $7 \%$ by weight), a soluble fiber abundant in apples, for 4 weeks reduced phospholipids excretion while increased bile acid excretion in feces compared to rats fed a control diet ( $0 \%$ by weight) [43]. Results suggest dietary soluble fibers increase the retention of phosphatidylcholine in the body, and thereby, provide a sufficient amount of choline for acetylcholine synthesis.

Other potential beneficial effects of soluble fiber intake have been suggested to be due to changes in the gut-brain axis [44]. Fermentation of soluble fibers by gut microbiota produces short-chain fatty acids (SCFAs): valeric, acetate, propionate, and butyrate [45]. In vitro, valeric acid and butyrate acid have been found to inhibit $\beta$-amyloid formation and aggregation in photoinduced cross-linking of unmodified proteins, suggesting SCFA attenuates the development of neurodegeneration [46].

To investigate the role of diet-induced microbiota composition on neuroinflammation, mice fed chow diet were microbiome depleted using antibiotics and then recolonized with microbiota harvested from the cecum and colon of either mice fed HFD (60\% total kcals) or mice fed a chow diet (13\% total kcals) [47]. Results showed a significant increase in microglial activation indicated by significantly increased protein expression of Iba1 and inflammatory 
markers, toll-like receptor 1 (TLR1) and TLR4 in the brain of mice fed HFD compared to chow diet. Based on the results microbiota modulation by HFD induces neuroinflammation in the brain. Another study investigating dietary effects on the vagus nerve connecting the gut to the CNS (nodose ganglion). Mice fed short-term (24h) HFD (60\% total kcals) had significantly increased Ibal protein expression and inflammatory marker TLR4 compared to mice fed a control diet (12\% total kcals) [48]. In addition, hypothalamic microglial activation was significantly higher in mice fed HFD compared to the control diet. Collectively, the study results supported an association between gut inflammation and hypothalamic neuroinflammation.

Another study investigated the consumption of dietary soluble fiber on SCFA levels and inflammation in aged mice since hypothalamic dysfunction and neurodegeneration diseases are induced due to chronic neuroinflammation. Lipopolysaccharide-treated aged (22-25 months) mice provided a high soluble fiber diet ( $5 \%$ inulin by weight) for 4 weeks altered gut microbiota composition and significantly increased SCFAs, butyrate, and acetate, compared to mice provided a low fiber diet (1\% cellulose by weight) [49]. Neuroinflammation measured as IL-1 $\beta$ and microglial activation were attenuated in aged mice fed high soluble fiber diet compared to low soluble fiber diet, suggesting anti-inflammatory effects of SCFAs in the brain. Similar findings were reported in a human clinical trial of patients with neurodegenerative diseases [50, 51]. Patients with Parkinson's disease had altered fecal microbiota composition and significantly lower SCFA concentration compared to healthy controls. The results suggested a role of microbiota and SCFAs in the pathology of neurodegenerative disease [50]. However, the study did not report dietary fiber consumption in the groups. 
Overall, the in vitro, preclinical and human studies reviewed reported consumption of soluble fibers increased choline for acetylcholine synthesis and ameliorated dysbiosis induced by consumption of the Western diet and these, in turn, reduced brain dysfunction, neuroinflammation, and development of neurogenerative diseases. In addition to soluble fiber, plant diets are also rich sources of polyphenols [4].

\section{Polyphenols on Brain Function and Neurodegeneration}

There is no RDA for dietary polyphenol intake. However, polyphenol consumption in the U.S. population is almost certainly low due to their low-dietary intake of fruits and vegetables, which are rich sources of polyphenols [52]. Polyphenol and their metabolites have antioxidant activity to neutralize reactive oxygen species (ROS) $[53,54]$. Chronic ROS also leads to mitochondrial dysfunction which has been implicated in decreased acetylcholine synthesis, a characteristic of neurodegenerative diseases [55]

Adult (aged 9-12 months) and older (aged 2-2.5 years) transgenic mice lacking ApoE were maintained on either a pro-oxidant diet deficient in folate and vitamin E (supplemented with $10 \%$ iron by diet weight, as a pro-oxidant) or a normal diet (4 mg/kg folic acid; $50 \mathrm{IU} / \mathrm{kg}$ Vit. E; 0\% supplemented iron) for 1 month [56]. Mice fed pro-oxidant diets were provided apple juice made from whole apples $(0.5 \% \mathrm{vol} / \mathrm{vol})$ or no apple juice (adjusted with sugar content) in drinking water. Results showed apple juice attenuated decreased brain acetylcholine content compared to a normal diet, suggesting apple juice antioxidant constituents, such as polyphenols, ameliorated a decrease in acetylcholine in the brain caused by pro-oxidant diet. 
Polyphenols were reported to attenuate ROS and mitochondrial dysfunction [57]. Rats (aged 4 months) were orally fed administered proanthocyanidins, a polyphenol abundant in grape seed extract, at 25,50 , and $75 \mathrm{mg} / \mathrm{kg} /$ day compared to a control diet ( $0 \mathrm{mg}$ ) for 9 weeks [57]. Brain oxidative stress was assessed by measuring superoxide dismutase and catalase activity as markers of antioxidants and malondialdehyde and protein carbonyl content as indicators for lipid peroxidation and protein oxidation. Brain dysfunction was assessed by measuring Chat and acetylcholinesterase activity and acetylcholine content. Rats fed $75 \mathrm{mg} / \mathrm{kg} / \mathrm{day}$ of proanthocyanidins showed significantly increased superoxide dismutase activity and decreased malondialdehyde and protein carbonyl content compared to control. Further, the brain acetylcholine content of rats fed $75 \mathrm{mg} / \mathrm{kg} /$ day of proanthocyanidins was significantly increased. This was accompanied by an increase in Chat activity and a decrease in acetylcholine esterase activity suggesting supplementation of proanthocyanidins attenuated ROS and neurodegeneration by increasing antioxidant activity and acetylcholine synthesis in the brain.

Grape seed proanthocyanidin extract has also been reported to exert other mechanisms. Mice fed a Western diet (35\% fat and 51\% carbohydrates kcal) supplemented with grape seed proanthocyanidins extract ( $25 \mathrm{mg} / \mathrm{kg}$ ) for 13 weeks ameliorated gene expression of POMC and phosphorylated STAT3 in the hypothalamus and reduced food intake compared to mice fed HFD. The results suggested leptin resistance in the hypothalamus was attenuated by grape seed proanthocyanidin extract intake [58].

Other studies showed polyphenols attenuated amyloidogenic pathway and the development of neurodegenerative diseases [59]. In vitro, resveratrol (trans-3,4',5trihydroxystilbene), an antioxidant polyphenol found in many plants, but particularly high in 
grapes, decreased $\beta$-amyloid proteins in HEK293 kidney cells transfected with human APP [59]. In an animal study, transgenic (APP/PS1) mice (aged 3 months old) were fed either standard chow diet or chow diets consisting of low (20 mg/kg/day) or high dose (40 mg/kg/day) quercetin, an antioxidant polyphenol abundant in grapes, for 16 weeks to investigate mitochondria, ROS production and senile plaques in the brain [53]. Results showed high-dose (40 mg/kg/day) quercetin significantly attenuated ROS production and increased adenosine triphosphate production compared to low-dose quercetin and chow fed mice. Furthermore, senile plaques were significantly decreased in the brain of mice fed the high-dose quercetin diet compared to low-dose quercetin [53]. The results suggested antioxidant properties of quercetin have the potential to lower the risk of age-related development of neurodegenerative diseases.

The effects of consuming grape powder rather than purified polyphenols on brain health were also investigated in vivo. Adult rats were fed a Western diet (50\% carbohydrate, $38 \%$ total kcals as fat) for 66 weeks to mimic the consumption of Western diet and to promote neurodegeneration. This was followed by supplementation with a low $(3.0 \% \mathrm{w} / \mathrm{w})$ or high $(6.0 \%$ w/w) dose of grape powder for 12 weeks [60]. Protein expression of the amylogenic pathway including $\beta$-secretase, $\alpha$-secretase, and $\beta$-amyloid was assessed in the brain [60]. At week 52, rats fed Western diet showed a significant increase in protein expression of $\beta$-secretase and $\beta$ amyloid accompanied by a decrease in $\alpha$-secretase compared to rats fed a control diet (76\% carbohydrate, $9 \%$ total kcals as fat). The results suggested Western diet consumption induced the formation of $\beta$-amyloid through the amyloidogenic pathway. In contrast, rats receiving a high dose $(6.0 \% \mathrm{w} / \mathrm{w})$ grape powder supplement for 12 weeks significantly attenuated protein expression of $\beta$-secretase and $\beta$-amyloid. This was accompanied by increased protein expression of $\alpha$-secretase. Results showed high dose grape powder intake attenuated the onset development 
of neurogenerative diseases by shifting the amyloidogenic pathway forming $\beta$-amyloid to the non-amyloidogenic pathway through the upregulation of $\alpha$-secretase protein expression.

Collectively, the studies reviewed support consumption of plant foods high in polyphenols such as fruits to counteract the negative impact on the brain caused by the Western diet. Polyphenols whether provided in purified form, as an extract or as powder were all found to have beneficial effects on brain health, suggesting consumption of fruits waste products has the potential to reduce the risk of neurodegeneration.

\section{Caloric Substitution of Western Diet with Apple Pomace}

Apples are one of the most popularly consumed fruits in the U.S. and a rich source of soluble fibers as well as polyphenols [61]. In a study comparing ten popularly consumed fruits in the U.S., apples ranked second in terms of total phenolic compounds (mg gallic acid equivalents/100g fruit) and total antioxidant activity ( $\mu$ mol vitamin $C$ equivalents/g fruit) [62]. The U.S. is the second global leader in apple production generating $~ 5,170,000$ metric tons of apples, annually [64]. Of the apple harvested, approximately $12 \%$ (620,400 metric tons) are processed into juice resulting in an estimated $25 \%(\sim 155,100$ metric tons) of the apple's mass being discarded as waste known as apple pomace [61, 64]. Apple pomace consists of skin, pulp, seeds, and stem [61].

Apple pomace's high water-activity (0.937) causes fermentation and microbial growth contributing to environmental pollution and the need for proper disposal at the cost of $\$ 10$ million, annually [61, 65]. Apple pomace is higher insoluble fibers (22\%), polyphenols $(0.029 \%)$, and fat (1.3-3.6\% predominantly linoleic acid and oleic acid) compared to a whole 
apple [61]. Based on nutrient composition, apple pomace has the potential to reduce the risk of neurogenerative diseases induced by Western diet consumption. This is important since an estimated 5.8 million Americans age 65 and older are living with Alzheimer's disease with an esitamted health care of $\$ 305$ billion and no effective treatments for attenuating disease progression [66]. The Alzheimer's Association recommends consuming diets high in fruits and vegetables while reducing SFAs and sugar to lower the risk of neurodegeneration. While constituents in apple pomace indicate potential use as a dietary therapy for preventing neurodegenerative diseases, apple pomace is high (12-22\%) in fructose compared to whole apples (5.8-6\%), which is a potential health concern [61, 67].

Fructose is a lipogenic substrate and has been implicated in the development of nonalcohol fatty liver disease (NAFLD) [68]. NAFLD is characterized by fat accumulation, increased liver inflammation, and increased risk of metabolic syndrome [68-69]. An estimated $\geq 25 \%$ of the U.S population has NAFLD [69]. NAFLD is reversible by diet and weight management [69]. However, untreated NAFLD leads to inflammation and progresses to nonalcoholic steatohepatitis (NASH), which has been implicated in liver failure and possibly neurodegeneration [69].

A study by Kim et al. [70] reported NAFLD accompanied by increased neuronal apoptosis, $\beta$-amyloid plaques, and microglial activation in the brain of transgenic mice (APP) fed HFD (37\% total kcal) compared to control diet (17\% total kcal) for 5 months. Switching transgenic mice consuming HFD to the control diet mid-study (3 months) decreased senile plaques in the brain compared to transgenic mice fed HFD until the end of the study (5 months) [70]. Results suggested NAFLD is implicated in neurodegeneration and attenuation by diet 
intervention, thus providing a rationale for caloric substitution of Western diet with apple pomace.

Skinner et al. [69] fed rats a Western diet (45\% fat total kcal) caloric substituted with $10 \%$ of apple pomace (Western/AP) found reduced fat infiltration in liver and downregulation of gene expression of diacylglycerol O-acyltransferase 2, an enzyme involved in DNL. Results suggested the fructose content in apple pomace had no significant effect on the pathology of NAFLD and NASH [69]. In a follow-up study, Skinner et al. [71] investigating Western/AP on gonadal adipose-liver crosstalk on inflammatory status. Results showed rats fed Western/AP attenuated gene expression of proinflammatory cytokines TNF- $\alpha$, IL- 6 and transcription factor, nuclear factor-kappa $\beta$, in gonadal adipose tissues, downregulated hepatic nuclear factor-kappa B gene expression, and increase serum total antioxidants [71]. Moreover, apple pomace mobilized palmitoleic acid (16:1n-7), oleic acid (18:1n-9), and palmitic acid (16:0) from the liver to gonadal adipose tissue [71]. Results suggested Western diet substitution with 10\% apple pomace reduced inflammation in adipose tissue and liver by decreasing lipid transport to the liver and by increasing serum antioxidant activity, which may have a similar effect on the hypothalamus.

Collectively, these animal studies provide a rationale for utilizing apple pomace as potential diet therapy for decreasing hypothalamic dysfunction and neurodegeneration. Plants require a copious amount of agricultural inputs such as irrigation water and the use of pesticides. Additionally, fruits and vegetables contribute to the highest total daily per capita of food waste (39\%) in the United States. Therefore, converting food waste to health-promoting sustainable foods can also contribute to reducing the environmental pollution. 
To our knowledge, no studies have investigated the effects of apple pomace on diet-induced hypothalamic dysfunction and neurodegeneration. Determining the effects of caloric substitution of Western diet with apple pomace on brain health as a potential sustainable functional food can contribute to reducing apple pomace disposal costs as well as health-care costs for treating neurodegenerative diseases. Therefore, studying the effects of caloric substitution of Western diet with apple pomace on diet-induced hypothalamic dysfunction and neurodegeneration is warranted. 


\section{References}

1- Camandola S, Mattson M. Brain Metabolism in Health, Aging, and Neurodegeneration. The EMBO Journal. 2017;36(11):1474-1492.

2- Bélanger M, Allaman I, Magistretti PJ. Brain Energy Metabolism: Focus on AstrocyteNeuron Metabolic Cooperation. Cell Metabolism. 2011;14:724-738.

3- Schönfeld P, Reiser G. Why does Brain Metabolism not Favor Burning of Fatty Acids to Provide Energy? - Reflections on Disadvantages of the Use of Free Fatty Acids as Fuel for Brain. Journal of Cerebral Blood Flow \& Metabolism. 2013;33:1493-1499.

4- Farooqui A, Farooqui T. Role of the Mediterranean Diet in the Brain and Neurodegenerative Diseases. 1st ed. OH, United States: Academic Press; 2018. p. 317-332.

5- Cai D, Liu T. Hypothalamic Inflammation: A Double-edged Sword to Nutritional Diseases. Annals of the New York Academy of Sciences. 2011;1243:E1-E39.

6- Xu AW, Kaelin CB, Takeda K, et al. PI3K Integrates the Action of Insulin and Leptin on Hypothalamic Neurons. The Journal of Clinical Investigation. 2005;115:951-958.

7- Varela L, Horvath TL. Leptin and Insulin Pathways in POMC and AgRP Neurons that Modulate Energy Balance and Glucose Homeostasis. EMBO reports. 2012;13:1079-1086.

8- Ahima RS, Antwi DA. Brain Regulation of Appetite and Satiety. Endocrinology and Metabolism Clinics of North America. 2008;37:811-823.

9- Vercruysse P, Vieau D, Blum D, et al. Hypothalamic Alterations in Neurodegenerative Diseases and Their Relation to Abnormal Energy Metabolism. Frontiers in Molecular Neuroscience. 2018;11:2.

10- Boleti AP de A, Flores TM de O, Moreno SE, et al. Neuroinflammation: An Overview of Neurodegenerative and Metabolic Diseases and of Biotechnological Studies. Neurochemistry International. 2020;136:104714.

11- Appendix 7. Nutritional Goals for Age-Sex Groups Based on Dietary Reference Intakes and Dietary Guidelines Recommendations - 2015-2020 Dietary Guidelines | health.gov [Internet]. Health.gov. 2020 [cited 2020 Jul 22]. Available from: https://health.gov/our-work/foodnutrition/2015-2020-dietary-guidelines/guidelines/appendix-7/

12- Bazinet RP, Layé S. Polyunsaturated Fatty Acids and their Metabolites in Brain Function and Disease. Nature Reviews Neuroscience. 2014;15:771-785.

13- Simopoulos AP. The Importance of the Omega-6/Omega-3 Fatty Acid Ratio in Cardiovascular Disease and Other Chronic Diseases. Experimental Biology and Medicine. 2008;233:674-688. 
14- Feingold, K.R. and C. Grunfeld, Introduction to Lipids and Lipoproteins, in Endotext, L.J. De Groot, et al., Editors. 2018: South Dartmouth (MA).

15- Ruíz-Gutiérrez V, Prada JL, Pérez-Jiménez F. Determination of Fatty Acid and Triacylglycerol Composition of Human Very-Low-Density Lipoproteins. Journal of Chromatography B: Biomedical Sciences and Applications. 1993;622:117-124.

16- Valdearcos M, Robblee MM, Benjamin DI, et al. Microglia Dictate the Impact of Saturated Fat Consumption on Hypothalamic Inflammation and Neuronal Function. Cell Reports. 2014;9:2124-2138.

17- Gimenez da Silva-Santi L, Masetto Antunes M, Mori MA, et al. Brain Fatty Acid Composition and Inflammation in Mice Fed with High-Carbohydrate Diet or High-Fat Diet. Nutrients. 2018;10:1277.

18- Cheng L, Yu Y, Szabo A, et al. Palmitic Acid Induces Central Leptin Resistance and Impairs Hepatic Glucose and Lipid Metabolism in Male Mice. The Journal of Nutritional Biochemistry. 2015;26:541-548.

19- Lin Bowen, Hasegawa Yu, Takane Koki, et al. High-Fat-Diet Intake Enhances Cerebral Amyloid Angiopathy and Cognitive Impairment in a Mouse Model of Alzheimer's Disease, Independently of Metabolic Disorders. Journal of the American Heart Association. 5:e003154.

20- Busquets O, Ettcheto M, Pallàs M, et al. Long-Term Exposition to a High Fat Diet Favors the Appearance of $\beta$-amyloid Depositions in the Brain of C57BL/6J Mice. A Potential Model of Sporadic Alzheimer's Disease. Mechanisms of Ageing and Development. 2017;162:38-45.

21- Maurer SV, Williams CL. The Cholinergic System Modulates Memory and Hippocampal Plasticity via Its Interactions with Non-Neuronal Cells. Frontiers in Immunology. 2017;8:1489.

22- Jeong JH, Lee DK, Jo Y-H. Cholinergic Neurons in the Dorsomedial Hypothalamus Regulate Food Intake. Molecular Metabolism. 2017;6:306-312.

23- Pedersen WA, Kloczewiak MA, Blusztajn JK. Amyloid Beta-protein Reduces Acetylcholine Synthesis in a Cell Line Derived From Cholinergic Neurons of the Basal Forebrain. Proceedings of the National Academy of Sciences. 1996;93:8068-8071.

24- Kaizer RR, da Silva AC, Morsch VM, et al. Diet-Induced Changes in AChE Activity after Long-Term Exposure. Neurochemical Research. 2004;29:2251-2255.

25- Bonda DJ, Stone JG, Torres SL, et al. Dysregulation of Leptin Signaling in Alzheimer Disease: Evidence for Neuronal Leptin Resistance. Journal of Neurochemistry. 2014;128:162-172. 
26- Ishii M, Iadecola C. Metabolic and Non-Cognitive Manifestations of Alzheimer's Disease:

The Hypothalamus as Both Culprit and Target of Pathology. Cell Metabolism. 2015;22:761776.

27- Gomes JAS, Silva JF, Marçal AP, et al. High-Refined Carbohydrate Diet Consumption Induces Neuroinflammation and Anxiety-Like Behavior in Mice. The Journal of Nutritional Biochemistry. 2020;77:108317.

28- Carta G, Murru E, Banni S, et al. Palmitic Acid: Physiological Role, Metabolism and Nutritional Implications. Frontiers in Physiology. 2017;8:902.

29- Butterworth RF. The Liver-Brain Axis in Liver Failure: Neuroinflammation and Encephalopathy. Nature Reviews Gastroenterology \& Hepatology. 2013;10:522-528.

30- Parimisetty A, Dorsemans A-C, Awada R, et al. Secret Talk Between Adipose Tissue and Central Nervous System via Secreted Factors-An Emerging Frontier in the Neurodegenerative Research. Journal of Neuroinflammation. 2016;13:67.

31- da Silva-Santi LG, Antunes MM, Caparroz-Assef SM, et al. Liver Fatty Acid Composition and Inflammation in Mice Fed with High-Carbohydrate Diet or High-Fat Diet. Nutrients. 2016;8:682.

32- Schmidt FM, Weschenfelder J, Sander C, et al. Inflammatory Cytokines in General and Central Obesity and Modulating Effects of Physical Activity. PLOS ONE. 2015;10:1-17.

33- Yeh SH-H, Shie F-S, Liu H-K, et al. A High-Sucrose diet Aggravates Alzheimer's Disease Pathology, Attenuates Hypothalamic Leptin Signaling, and Impairs Food-Anticipatory Activity in APPswe/PS1dE9 Mice. Neurobiology of Aging. 2020;90:60-74.

34- Stephan BCM, Wells JCK, Brayne C, et al. Increased Fructose Intake as a Risk Factor For Dementia. The Journals of Gerontology: Series A. 2010;65A:809-814.

35- Erbas O, Erdogan MA, Khalilnezhad A, et al. Neurobehavioral Effects of Long-Term Maternal Fructose Intake in Rat Offspring. International Journal of Developmental Neuroscience. 2018;69:68-79.

36- Harrell CS, Zainaldin C, McFarlane D, et al. High-Fructose Diet During Adolescent Development Increases Neuroinflammation and Depressive-like Behavior Without Exacerbating Outcomes After Stroke. Brain, Behavior, and Immunity. 2018;73:340-351.

37- M. Katherine Hoy, EdD, RD, Joseph D. Goldman, MA. Fiber Intake of the U.S. Population What We Eat in America, NHANES 2009-2010 [Internet]. [cited 2019Mar6]. Available from: https://www.ars.usda.gov/ARSUserFiles/80400530/pdf/DBrief/12_fiber_intake_0910.pdf

38- Palafox-Carlos H, Ayala-Zavala JF, González-Aguilar GA. The Role of Dietary Fiber in the Bioaccessibility and Bioavailability of Fruit and Vegetable Antioxidants. Journal of Food Science. 2011;76:R6-R15. 
39- Jenkins D, Wolever T, Rao AV, et al. Effect on Blood Lipids of Very High Intakes of Fiber in Diets Low in Saturated Fat and Cholesterol. New England Journal of Medicine. 1993;329:21-26.

40- Alvaro D, Cantafora A, Attili AF, et al. Relationships Between Bile Salts Hydrophilicity and Phospholipid Composition in Bile of Various Animal Species. Comparative Biochemistry and Physiology Part B: Comparative Biochemistry. 1986;83:551-554.

41- Chung S-Y, Moriyama T, Uezu E, et al. Administration of Phosphatidylcholine Increases Brain Acetylcholine Concentration and Improves Memory in Mice with Dementia. The Journal of Nutrition. 1995;125:1484-1489.

42- Kritchevskya D, Tepper SA, Satchithanandam S, et al. Dietary Fiber Supplements: Effects on Serum and Liver Lipids and on Liver Phospholipid Composition in Rats. Lipids. 1988;23:318-321.

43- Garcia-Diez F, Garcia-Mediavilla V, Bayon JE, et al. Pectin Feeding Influences Fecal Bile Acid Excretion, Hepatic Bile Acid and Cholesterol Synthesis and Serum Cholesterol in Rats. The Journal of Nutrition. 1996;126:1766-1771.

44- Noble EE, Hsu TM, Kanoski SE. Gut to Brain Dysbiosis: Mechanisms Linking Western Diet Consumption, the Microbiome, and Cognitive Impairment. Frontiers in Behavioral Neuroscience. 2017;11:9.

45- Silva YP, Bernardi A, Frozza RL. The Role of Short-Chain Fatty Acids From Gut Microbiota in Gut-Brain Communication. Frontiers in Endocrinology. 2020;11:25.

46- Ho L, Ono K, Tsuji M, et al. Protective Roles of Intestinal Microbiota Derived Short Chain Fatty Acids in Alzheimer's Disease-Type Beta-amyloid Neuropathological Mechanisms. Expert Review of Neurotherapeutics. 2018;18:83-90.

47- Bruce-Keller AJ, Salbaum JM, Luo M, et al. Obese-type Gut Microbiota Induce Neurobehavioral Changes in the Absence of Obesity. Biological Psychiatry. 2015;77:607615.

48- Waise TMZ, Toshinai K, Naznin F, et al. One-day High-Fat Diet Induces Inflammation in the Nodose Ganglion and Hypothalamus of Mice. Biochemical and Biophysical Research Communications. 2015;464:1157-1162.

49- Matt SM, Allen JM, Lawson MA, et al. Butyrate and Dietary Soluble Fiber Improve Neuroinflammation Associated With Aging in Mice. Frontiers in Immunology. 2018;9:1832.

50- Unger MM, Spiegel J, Dillmann K-U, et al. Short Chain Fatty Acids and Gut Microbiota Differ Between Patients with Parkinson's Disease and Age-matched Controls. Parkinsonism \& Related Disorders. 2016;32:66-72.

51- Pluta R, Ułamek-Kozioł M, Januszewski S, et al. Gut Microbiota and Pro/Prebiotics in Alzheimer's Disease. Aging (Albany NY). 2020/03/19 ed. 2020;12:5539-5550. 
52- Dietary Intakes Compared to Recommendations. Percent of the U.S. Population Ages 1 Year \& Older Who Are Below, At, or Above Each Dietary Goal or Limit [Internet]. DIETARY GUIDELINES FOR AMERICANS 2015-2020. [cited 2019Mar17]. Available from: https://health.gov/dietaryguidelines/2015/resources/2015-2020_dietary_guidelines.pdf

53- Wang D-M, Li S-Q, Wu W-L, et al. Effects of Long-term Treatment with Quercetin on Cognition and Mitochondrial Function in a Mouse Model of Alzheimer's Disease.

Neurochemical Research. 2014;39:1533-1543.

54- Sarubbo F, Moranta D, Pani G. Dietary Polyphenols and Neurogenesis: Molecular Interactions and Implication for Brain Ageing and Cognition. Neuroscience \& Biobehavioral Reviews. 2018;90:456-470.

55- Guo L, Tian J, Du H. Mitochondrial Dysfunction and Synaptic Transmission Failure in Alzheimer's Disease. J Alzheimers Dis. 2017;57:1071-1086.

56- Chan A, Graves V, Shea TB. Apple Juice Concentrate Maintains Acetylcholine Levels Following Dietary Compromise. Journal of Alzheimer's Disease. 2006;9:287-291.

57- Devi A, Jolitha AB, Ishii N. Grape Seed Proanthocyanidin Extract (GSPE) and Antioxidant Defense in the Brain of Adult Rats. Medical science monitor : international medical journal of experimental and clinical research. 2006;12:BR124-9.

58- Ibars M, Ardid-Ruiz A, Suárez M, et al. Proanthocyanidins Potentiate Hypothalamic Leptin/STAT3 Signaling and POMC Gene Expression in Rats with Diet-induced Obesity. Int J Obes (Lond). 2016/09/28 ed. 2017;41:129-136.

59- Marambaud P, Zhao H, Davies P. Resveratrol Promotes Clearance of Alzheimer's Disease Amyloid- $\beta$ Peptides. Journal of Biological Chemistry. 2005;280:37377-37382.

60- Chou L-M, Lin C-I, Chen Y-H, et al. A Diet Containing Grape Powder Ameliorates the Cognitive Decline in Aged Rats with a Long-term High-Fructose-High-Fat Dietary Pattern. The Journal of Nutritional Biochemistry. 2016;34:52-60.

61- Skinner RC, Gigliotti JC, Ku K-M, et al. A Comprehensive Analysis of the Composition, Health Benefits, and Safety of Apple Pomace. Nutrition Reviews. 2018;76:893-909.

62-Sun J, Chu Y-F, Wu X, et al. Antioxidant and Antiproliferative Activities of Common Fruits. Journal of Agricultural and Food Chemistry. 2002;50:7449-7454.

63- USDA/NASS Apple Production Measured in LB [Internet]. Quickstats.nass.usda.gov. 2020 [cited 2020 Aug 3]. Available from: https://quickstats.nass.usda.gov/results/2747ED7932EC-3268-8E8E-015C6A49B6DB

64- USDA/NASS Apple Processing Juice Production Measured in LB [Internet]. Quickstats.nass.usda.gov. 2020 [cited 2020 Aug 3]. Available from: https://quickstats.nass.usda.gov/results/15455AF2-7E21-3FC0-BAB7-DA2247C9901D 
65- Vendruscolo F, Albuquerque PM, Streit F, et al. Apple Pomace: A Versatile Substrate for Biotechnological Applications. Critical Reviews in Biotechnology. 2008;28:1-12.

66- (2020). 2020 Alzheimer's Disease Facts and Figures. Alzheimer's \& Dementia. 2020;16:391-460.

67- Sato MF, Vieira RG, Zardo DM, et al. Apple Pomace From Eleven Cultivars: An Approach to Identify Sources of Bioactive Compounds. Acta Scientiarum Agronomy. 2010;32:29-35.

68- Mock K, Lateef S, Benedito VA, et al. High-Fructose Corn Syrup-55 Consumption Alters Hepatic Lipid Metabolism and Promotes Triglyceride Accumulation. The Journal of Nutritional Biochemistry. 2017;39:32-39.

69- Skinner RC, Warren DC, Lateef SN, et al. Apple Pomace Consumption Favorably Alters Hepatic Lipid Metabolism in Young Female Sprague-Dawley Rats Fed a Western Diet. Nutrients. 2018;10:1882.

70- Kim DG, Krenz A, Toussaint LE, et al. Non-Alcoholic Fatty Liver Disease Induces Signs of Alzheimer's Disease (AD) in Wild-Type Mice and Accelerates Pathological Signs of AD in an AD Model. Journal of Neuroinflammation. 2016;13:1.

71- Skinner RC, Warren DC, Naveed M, et al. Apple Pomace Improves Liver and Adipose Inflammatory and Antioxidant Status in Young Female Rats Consuming a Western Diet. Journal of Functional Foods. 2019;61:103471. 


\section{Chapter 2}

\section{Study Objective and Hypothesis}

Objective: To determine the effects of Western diet and caloric substitution of Western diet with $10 \%$ apple pomace on brain fatty acid composition and expression of genes related to hypothalamic function and neurodegeneration risk in growing female rats.

Hypothesis: Caloric substitution of Western diet with $10 \%$ of apple pomace will change fatty acids composition in the brain, and this in turn, will attenuate expression of genes related to hypothalamic dysfunction and neurodegeneration in growing female rats. 


\title{
Chapter 3
}

Apple Pomace Favorably Alters Brain Fatty Acid Composition and Gene Expression in the Hypothalamus of Growing Female Sprague-Dawley Rats Fed a Western Diet

\author{
Ayad A. Alawadi ${ }^{a}$, Derek C. Warren ${ }^{b}$ Nariman A. Ktil $^{\mathrm{a}}$, R. Chris Skinner ${ }^{\mathrm{b}}$, Vagner A. Benedito ${ }^{\mathrm{c}}$ \\ and Janet C. Tou ${ }^{\mathrm{a}^{*}}$, \\ ${ }^{a}$ Division of Animal and Nutritional Sciences, West Virginia University, Morgantown, WV \\ 26506, United States \\ ${ }^{b}$ Division of Natural Sciences and Mathematics, University of Ozarks, Clarksville, AR, 72830, \\ United States \\ ${ }^{c}$ Division of Plant and Soil Sciences, West Virginia University, Morgantown, WV 26506, United \\ States
}

*Corresponding author:

Janet C. Tou, PhD

Human Nutrition and Foods

e-mail: janet.tou@mail.wvu.edu

Target journal: Nutritional Neuroscience 


\begin{abstract}
Background: The hypothalamus plays a major role in regulating food-intake and energyexpenditure. Hypothalamic dysfunction implicated in neurodegenerative diseases is characterized by diet-induced neuroinflammation and energy dysregulation. Apple pomace, a waste byproduct of processing, is rich in polyphenols and soluble fibers, has the potential to ameliorate diet-inducing inflammation.
\end{abstract}

Objective: The objective of this study was to determine the effects of Western diet and caloric substitution of Western diet with $10 \%$ apple pomace on brain fatty acid composition and gene expression related to hypothalamic function and health in growing female rats.

Methods: Growing female Sprague-Dawley rats (age 22-29 days) were randomly assigned ( $\mathrm{n}=8$ rats/group) to consume purified AIN-93G (control), Western diet, or Western calorically substituted with $10 \%$ apple pomace (Western/AP) diets for 8 weeks. Brain lipid content and fatty acid profile analysis were measured. Differentially expressed genes were measured in the hypothalamus using RNA-Seq.

Results: Western/AP diet consisted of the highest amount of soluble fibers and polyphenols. Brain oleic acid was highest in rats fed Western/AP diet $(p<0.0005)$. RNA-seq results comparing the hypothalamus of rats fed Western to Western/AP showed 15 differentially expressed genes, of which 5 genes: phospholipase D family member 5 (PLD5), synuclein alpha (Snca), NADH dehydrogenase (ubiquinone) Fe-S protein 6 (Ndufs6), choline O-acetyltransferase (Chat), and frizzled class receptor 6 (Fzd6) were implicated in neurodegenerative diseases. Apple pomace attenuated the upregulation of Snca $(q<0.05)$, Chat $(q<0.05)$, and Ndufs6 $(q<0.05)$, as well as downregulated PLD5 $(q<0.06)$, and Fzd6 $(q<0.05)$ gene expression. Results suggested apple pomace dietary supplementation led to reduced neurodegeneration, 
acetylcholine impairment, and mitochondrial dysfunction through the modulation of brain lipid content and profile.

Conclusion: The results provide evidence that partial caloric substitution of Western diet with apple pomace has the potential to attenuate the pathogenesis of neurodegenerative diseases.

Significance: Based on pre-clinical evidence, apple pomace has the potential to be a sustainable functional food for brain health.

Keywords: Western diet, apple pomace, hypothalamus, neurodegenerative diseases, functional foods. 


\section{Introduction}

The hypothalamus orchestrates signals from the central nervous system (CNS) and regulates basic body functions that include a major role in regulating food intake and energy expenditure [1]. In the hypothalamus, the upregulation of Agouti-related protein neurons (AgRP) by the lack of binding of both neuroendocrine signals, leptin, and insulin, to their receptors induce orexigenic effects [1], whereas the upregulation of Pro-opiomelanocortin neurons (POMC) induces anorexigenic effects in the hypothalamus [1]. Disruption, such as inflammation, in hypothalamic neurons controlling satiety and appetite, leads to imbalances in energy homeostasis as well as the development of metabolic syndromes and neurodegenerative diseases [2].

Accumulation of misfolded proteins in the hypothalamus caused by neuroinflammation results in the onset of neurodegenerative diseases, including Alzheimer's and Parkinson's diseases [3]. The impairment of cholinergic neurons is one of the hallmarks of neurodegenerative diseases, possibly due to the reduction of the neurotransmitter acetylcholine, which has implications in modulating POMC neurons in the hypothalamus $[4,5]$. Choline is a substrate in the de novo synthesis of acetylcholine and the liver is the central organ responsible for choline metabolism [6]. Patients with non-alcoholic fatty liver disease (NAFLD) have reported being deficient in choline, suggesting a relationship between diet-induced NAFLD and neurodegeneration in the CNS [6]. Kim et al. [7] reported NAFLD was accompanied by increased neurodegeneration in mice fed a Western-style diet.

The Western diet is characterized by calorically dense foods high in refined sugars (e.g. sucrose and fructose) and fat (e.g. long-chain saturated fatty acids >16C), but low in protein, soluble fibers, and polyphenols [8]. Studies have reported diets high in fat and sugar 
induced changes in brain lipid composition and food intake implicated in neurodegeneration [9].

In 2020, an estimated 5.8 million Americans age $\geq 65$ were diagnosed with Alzheimer's disease with an estimated health care costs of $\$ 305$ billion [10]. The Alzheimer's Association recommends consuming diets high in fruits and vegetables while reducing saturated fatty acids and refined sugars to lower the risk of neurodegeneration [10]. Apple pomace consists mainly of skin, pulp, seeds, and stem makes it a rich source of soluble fibers and polyphenols. Nutrient analysis report apple pomace is low in fat (1.3-3.6\%: mostly as linoleic acid and oleic acid), high in soluble fibers (22\%), and antioxidant polyphenols $(0.029 \%)[8,11]$. However, apple pomace is also high in fructose. Previously, our laboratory found growing rats fed a Western diet (45\% fat total kcal) substituted with $10 \%$ of apple pomace attenuated the development of NAFLD [11]. Given reports of NAFLD and risk of neurodegeneration, this led us to hypothesize that apple pomace has the potential to attenuate hypothalamus dysfunction and neurodegeneration induced by a Western diet.

An issue of emerging importance in the field of nutrition and food sciences is converting food waste into sustainable foods that reduce strain on the environment while improving dietquality and promoting a healthy population. Although rich in nutrients, apple pomace is considered a waste product of apple processing which is costly for producers to dispose of as well as adds to environmental pollution $[8,11]$. Fruits and vegetables contribute to the highest amount of total food waste daily per capita (39\%) in the United States [12].

To our knowledge, no studies have investigated the effects of apple pomace on hypothalamic dysfunction related to neurogenerative diseases. Using RNA sequencing technology, we performed an exploratory study on the rat brain in order to determine the effects 
of Western diet caloric substitution with apple pomace on global expression of genes implicated in food intake and neurodegeneration.

\section{Materials and Methods}

\section{Animals and Diets}

All animal procedures used in this study were approved by Animal Care and Use Committee at West Virginia University and were conducted according to the National Research Council for the Care of Laboratory Animal Guidelines. Female Sprague-Dawley rats (age 22-29 days) were obtained from Harlan-Teklad (Indianapolis, IN, USA). Upon arrival, rats were individually housed in metabolic cages and kept in a room at a temperature of $21 \pm 2^{\circ} \mathrm{C}$ with a 12 $\mathrm{h}$ light/dark cycle.

At the end of 7 days of acclimation, rats were randomly assigned to three dietary groups ( $\mathrm{n}=8$ rats/group) consisting of (1) the American Institute of Nutrition-93G (AIN) (3.8 kcal/g), a standard purified rodent diet formulated to meet the nutrient requirements of growing animals as a control, (2) Western diet (45\% saturated fat, 33\% sucrose by kcals), or (3) Western diet with $10 \%$ of diet weight $(\mathrm{g} / \mathrm{kg}$ ) substituted with apple pomace (Western/AP). Apple pomace was provided by Swilled Dog Hard Cider Company (Franklin, WV, USA). Nutrient analysis of the apple pomace was performed by Medallion Laboratories (Minneapolis, MN, USA), and total polyphenols determined using the Folin-Ciocalteu method [13]. Western diet and Western diets substituted with $10 \%$ apple pomace were adjusted to be isocaloric by adjusting fat and carbohydrates $\mathrm{kcal}\left(4.7 \mathrm{kcal} / \mathrm{g}\right.$ ) (Table 1). All experimental diets were stored at $-20^{\circ} \mathrm{C}$ until fed to rats. 
Rats were given ad libitum access to their randomly assigned diet and deionized water $\left(\mathrm{ddH}_{2} \mathrm{O}\right)$ throughout the 8 weeks study duration. Food intake was measured daily and replaced with assigned fresh diets. $\mathrm{ddH}_{2} \mathrm{O}$ and body weight was measured weekly. Feed Efficiency Ratio was calculated by weight gain $(\mathrm{g})$ divided by food intake $(\mathrm{g}) * 100$. At the end of 8 weeks, rats were fasted overnight then euthanized by carbon dioxide inhalation. Brain was excised, weighed, and then flash-frozen in liquid nitrogen and stored at $-80^{\circ} \mathrm{C}$ until analyzed.

\section{Brain Total Lipid Content and Fatty Acid Composition}

Lipid extraction was performed on brain tissue according to Bligh and Dyer [14]. Briefly, 0.1g of brain tissue was homogenized in Tris/EDTA buffer (pH 7.4). Nonadecanoic acid (19:0; $50 \mu \mathrm{L}$ ) was added as a standard during the initial weighing of the samples to quantify fatty acids. Chloroform: methanol: acetic acid (2:1:0.15,v/v/v) solution was added to brain samples, centrifuged at $900 \times g$ for $10 \mathrm{~min}$ at $10^{\circ} \mathrm{C}$, and then the bottom chloroform layer collected. The collected chloroform layer was mixed with chloroform: methanol $(4: 1 \mathrm{v} / \mathrm{v})$, and centrifuged at $900 \times g$ at $10^{\circ} \mathrm{C}$ for $10 \mathrm{~min}$. The chloroform layer was collected and filtered by phase separation filters. Extracted lipids were dried under nitrogen gas. Total lipid content in the brain was determined gravimetrically.

Following lipid extraction, brain tissue samples were transmethylated according to the method described by Fritsche and Johnston [15]. Briefly, fatty acids were methylated by adding $4 \%$ sulfuric acid in anhydrous methanol to the extracted lipid samples followed by incubation in a $90^{\circ} \mathrm{C}$ water bath for $60 \mathrm{~min}$. Samples were cooled to room temperature and $\mathrm{ddH}_{2} \mathrm{O}$ were added. Chloroform was added to the methylated samples and then centrifuged at $900 \times g$ for $10 \mathrm{~min}$ at $10^{\circ} \mathrm{C}$. The collected chloroform layer was filtered through anhydrous sodium sulfate to remove 
any remaining water. Fatty acid methyl esters (FAMEs) were dried under nitrogen gas and resuspended in iso-octane.

FAMEs were analyzed by gas liquid chromatography (GLC) (model CP-3800; Varian, Walnut Creek, CA, USA) using an initial temperature of $140^{\circ} \mathrm{C}$ held for 5 min and then increased $1^{\circ} \mathrm{C}$ per min to a final temperature of $220^{\circ} \mathrm{C}$. A wall-coated open tubular fused silica capillary column (Varian, Walnut Creek, CA, USA) was used to separate FAME with CP-Sil 88 at the stationary phase. Nitrogen was used as the carrier gas and the total separation time was 56 min. Quantitative 37 Component FAMEs Sigma Mix (Supelco, Bellefonte, PA, USA) was used to identify fatty acids. Fatty acids were determined by retention time and quantified using peak counts. All samples were performed in duplicate and reported as $\%$ of total fatty acids.

\section{RNA Isolation and Sequencing}

Shown in Figure 1, a flowchart of RNA-seq study and data analysis. Under aseptic conditions, the hypothalamus was excised from frozen brain tissue using a sterile $5 \mathrm{~mm}$ biopsy tool based on rat brain Atlas [16]. Total RNA was extracted from the hypothalamus according to the manufacturer's instruction using the mirVana miRNA Isolation Kit (Thermo Scientific, Waltham, MA, USA). Following DNase I treatment with BIO-RAD kit (Hercules, CA, USA), isolated RNA integrity was quantified by spectrophotometry (NanoDrop 100; Thermo Scientific, Waltham, MA, USA). RNA quality was determined by Agilent 2100 Bioanalyzer. mRNA samples $\geq 9.0$ RNA Integrity Number (RIN) were used to construct sequencing libraries ( $\mathrm{n}=5$ per group). Library preparation was performed by commercially available Novogene (Sacramento, CA, USA) using Illumina 1.9 HiSeq resulting in 19-22 million clean reads per sample and generation of $150 \mathrm{bp}$ paired-end reads. Raw sequenced reads were filtered out for low quality and adapters. All samples ( $\mathrm{n}=30$ left/right) had an effective rate greater than $93.50 \%$ (Clean 
reads/Raw reads*100\%). Sample reads were mapped to their input read with an average of $93.9 \%$.

RNA-Seq Data Analysis and Quality Control

All sample paired-reads FASTQ files were quality checked using Fastqc (version 0.11.5). This produced sequencing files that have an average per base Phred score $>35$, which indicates a high-quality base call. VICE DESeq2 pipeline was used to analyze gene expression through Cyverse Discovery Environment (de.cyverse.org) [17]. RMTA v2.6.3 and Rstudio-DESeq2 app were used to determine gene expression according to the pipeline workflow. Rattus norvegicus (GCF_000001895.5/ Rnor_6.0) genome assembly was used as a reference [18]. Gene annotation (Rattus norvegicus) Rnor_6.0.99.gtf.gz was obtained from the Ensembl website (useast.ensembl.org) and used to annotate the genome assembly. HISAT2 was used to map samples reads to the genome assembly, according to the pipeline, producing feature counts [17]. Principle component analysis graph was produced by pcaexplorer [19]. Gene upregulation and downregulation was visualized by heatmaps produced using R studio (version 1.2.5033) by ggplot2 and pheatmap functions. Phenotypes of differentially expressed genes were obtained from the Ensembl BioMart tool filtered based on gene ID, gene name, and phenotype description [20]. PANTHER expression analysis tool was used to determine gene pathway association [21].

\section{Statistical Analysis}

Statistical analyses for non-RNA-seq experiments were performed using JMP 12.2 statistical software package (SAS Institute, Cary, NC, USA). Results are expressed as mean \pm standard error of the mean (SEM). Data were analyzed for normal distribution and homogeneity of variance prior to conducting One-Way Analysis of Variance (ANOVA) to determine the differences among dietary groups. Post hoc multiple comparison tests were performed on 
parametric data using Tukey's test with differences considered to be significant at $p<0.05$. For gene expression analysis, feature counts were normalized using shifted logarithm transformation and visualized by VICE DESeq2 pipeline script [17]. Significantly different gene expressions were determined based on adjusted p-value less than $0.10(q<0.10)$ according to the DESeq2 script.

\section{Results}

Diet analysis

Caloric substitution of Western diet with $10 \%$ apple pomace (Western/AP) resulted in the highest polyphenol content among experimental diets. All diets were adjusted for 5\% of total dietary fiber. Western and AIN diets consisted of 5\% insoluble fiber (cellulose); whereas, Western/AP diet consisted of 3.9\% insoluble fiber (cellulose) and 1.1\% soluble fiber (pectin). Western/AP diet had higher fructose (17.5\%) content compared to Western diet (17\%) and AIN diet $(10 \%)$. Western diet had higher sucrose (34\%) content compared to Western/AP (28.4\%) and AIN (10\%) diet. Both Western diets had higher fat content (45\% total kcal) than AIN diet (17\% total kcal) (Table 1).

Table 2 shows the fatty acid profile of experimental diets. Western/AP had the highest palmitic acid content $(p<0.05)$ among experimental diets. Western diets were higher $(p<0.05)$ in saturated fatty acids (SFAs), palmitic acid (16:0), and stearic acid (18:0) compared to AIN diet. Additionally, Western diets were higher $(p<0.05)$ in monounsaturated fatty acids (MUFAs), palmitoleic acid (16:1n-7), and oleic acid (18:1n-9), but significantly lower in essential fatty acids, $\alpha$-linolenic acid (18:3n-3) and linoleic acid (18:2n-6) compared to AIN diet. The long-chain omega-6 polyunsaturated fatty acid (PUFAs), arachidonic acid (ARA, 20:4n-6) was higher $(p<0.05)$ in Western diets compared to the AIN diet. In all diets, long-chain omega- 
3 polyunsaturated fatty acids, eicosapentaenoic acid (EPA), and docosahexaenoic acid (DHA) were below the GLC detection level.

Food intake, Body Weight and Adiposity

Total caloric intake was higher $(p<0.0006)$ in rats fed Western diets compared to rats fed AIN diet. Lipid intake was higher $(p<0.0001)$ in rats fed Western diets compared to rats fed AIN diet. Total carbohydrate intake was lower in rats fed Western diets compared to rats fed AIN diet $(p<0.0001)$. There was no significant difference in lipid and total carbohydrate intake between rats fed Western diet compared to Western/AP. There were no significant differences in total protein intake and feed efficiency ratio among rats fed different experimental diets (Table 3).

At the end of the 8 weeks feeding study, rats fed Western/AP diet, but not Western diet had higher $(p<0.0373)$ final body weight compared to rats fed the AIN diet. Gonadal fat pad weight was significantly greater $(p<0.0022)$ in rats fed Western diets compared to AIN diet. There were no significant differences in brain weight expressed as absolute or relative weight among rats fed different experimental diets (Table 3).

\section{Total Brain Lipid Content and Fatty Acid Composition}

Shown in Table 4, rats fed Western diet had the highest $(p<0.0001)$ brain lipid content. Brain lipid content was not significantly different in rats fed Western/AP compared to AIN diet. Rats fed Western/AP had the highest $(p<0.0005)$ brain oleic acid $(18: 1 \mathrm{n}-9)$. Rats fed Western/AP, but not Western diet had lower $(p<0.0297)$ brain linoleic acid $(18: 2 n-6)$ compared to rats fed AIN diet. There were no significant differences in palmitic acid (16:0), stearic acid (18:0), and DHA (22:6n-3) composition in the brain of rats fed any of the experimental diets. 
Palmitoleic acid (16:1n-7), $\alpha$-linolenic acid (18:3n-3), ARA (20:4n-6), and EPA (20:5n-3) in brain tissue were below the GLC detection level.

\section{Global Hypothalamic Gene Expression Analysis}

In Figure 2, global principal component analysis (PCA) showing random scatter points of all rats fed different experimental diets, indicated no clear differences in gene expression in the hypothalamus. Global hierarchical clustering analysis (HCA) heatmap of rats fed different experimental diets showed similar color shadings across all samples as well as random column clustering denoting no significant differences in gene expression in the hypothalamus (Figure 3). Likewise, HCA heatmap of genes implicated in food intake pathway regulating POMC and AgRP neurons which included: leptin receptors (LepR), insulin receptors (InsR), janus kinase 3 (Jak3), signal transducer and activator of transcription 3 (STAT3), forkhead box protein O1 (FOXO1), phosphoinositide 3-kinases (Pi3k), cocaine-and amphetamine-regulated transcript (Cart), neuropeptide Y (Npy), and melanocortin 4 receptor (Mc4r) also showed no significant differences in gene expression in the hypothalamus within groups donated by similar color shading across all samples as well as random column clustering (Figure 4).

Gene expression analysis of the hypothalamus of rats fed Western diets compared to AIN diet resulted in two differentially expressed genes in the hypothalamus. Fas apoptotic inhibitory molecule (Faim) was downregulated $(q<0.005)$ and coiled-coil domain-containing protein 72 like gene expression was upregulated $(q<0.0005)$ in rats fed Western and Western/AP compared to AIN diet (Table 5).

Western diets Hypothalamic Gene Expression Analysis

Comparison of rats fed Western/AP diet to rats fed Western diet resulted in 15 differentially expressed genes $(q<0.10)$ (Table 5). HCA heatmap of the 15 genes expressed 
showed different color shading and similar column clustering of each rat fed Western/AP compared to Western diet, indicating differences between the groups (Figure 5). The eight differentially upregulated genes in rats fed Western/AP compared to Western diet were: 1) centrin-3-like (LOC100912538), 2) microRNA 2964 (Mir2964), 3) frizzled class receptor 6 (Fzd6), 4) solute carrier family 35 member F3 (Slc35f3), 5) heparan sulfate-glucosamine 3sulfotransferase 1 (Hs3st1), 6) ring finger FYVE-like ligase (Rffl), 7) inositol polyphosphate-5phosphatase $\mathrm{F}$ (Inpp5f), and 8) phospholipase D family, member 5 (Pld5) (Figure 6).

The seven genes differentially downregulated in rats fed Western/AP compared to rats fed Western diet were:1) NBL1, DAN family BMP antagonist (Nbl1), 2) synuclein alpha (Snca), 3) insulin-like growth factor binding protein 4 (Igfbp4), 4) choline O-acetyltransferase (Chat), 5) cyclin T1 (Ccnt1), 6) NADH dehydrogenase (ubiquinone) Fe-S protein 6 (Ndufs6), and 7) Von Willebrand factor A domain-containing 5A (Vwa5a) (Figure 6). Shown in Table 6 are phenotype, disease, and trait of differentially expressed genes in the hypothalamus of rats fed Western/AP compared to Western diet.

\section{Discussion}

Studies on high-fat diet consumption showed dietary fatty acids accumulate directly in the brain $[22,23]$. Taha et al. $[24,25]$ reported lower linoleic acid (18:2n-6) intake in rats attenuated lipopolysaccharide-induced neuroinflammation by reducing metabolism to ARA (20:4n-6). In our study ARA (20:4n-6) concentration was below the GLC detection level; however, rats fed Western/AP, but not Western diet despite no differences in diet content had significantly lower brain linoleic acid (18:2n-6) compared to rats fed AIN diet. Caloric substitution with apple pomace may have reduced brain linoleic acid (18:2n-6) concentration due to its high pectin content. Dietary soluble fibers have been reported to bind and decrease fatty 
acid absorption [26]. Western diets are high in fats particularly, SFAs. Increased brain lipid content has been found to promote neurodegeneration and SFAs have been suggested to induce neuroinflammation $[22,23]$. Our study found the highest brain lipid content in rats fed Western diet but no significant difference in brain SFAs, palmitic (16:0), and stearic (18:0) acid, among diet groups. In a study of mice administered various purified fatty acids, palmitic acid (16:0), but not oleic acid (18:1n-9), activated microglial cells and inflammation in the hypothalamus [23].

Oleic acid (18:1n-9) is a major component of membrane phospholipids and myelin in neuronal cells and a neurotrophic factor promoting axonal, dendritic, and synapse formation [27]. In a postmortem study of humans with Parkinson's and Alzheimer's disease, oleic acid content in the brain was lower compared to age-matched healthy controls [28]. Previously, we reported rats fed Western/AP diet increases the deposition of oleic acid from the liver to gonadal tissues and attenuated hepatic inflammation [29]; this led us to hypothesize that similar effects may also occur on the brain. In the present study, rats fed Western/AP had the highest brain oleic acid (18:1n-9) concentration despite no differences in the oleic acid content of the diets. This suggested caloric substitution with $10 \%$ of apple pomace may have resulted in the mobilization of oleic acid (18:1n-9) from the liver to the brain. Altered brain fatty acid profile has important implications since fatty acids have been reported to stimulate gene expression, neuronal activity synaptogenesis, and neurogenesis, and to prevent neuroinflammation and apoptosis [30]. To investigate altered brain function and neurodegeneration risk induced by altered lipid brain profile, we used RNA-Seq to explore global gene expression in the hypothalamus.

The hypothalamus regulates various functions, including key roles in regulating food intake and energy expenditure [1]. Hypothalamic global gene expression showed minor differences indicated by PCA and HCA among dietary groups (Figure 2-4). Comparison of rats 
fed Western diets to AIN-fed rats resulted in only two differentially expressed genes, fas apoptotic inhibitory molecule (Faim) and coiled-coil domain-containing protein 72-like (Table 5). To our knowledge, no studies have found a role of coiled-coil domain-containing protein 72 like in the CNS while Faim has been reported to protect neural cells from apoptosis and promote neurite growth in the CNS $[31,32,33]$. In our study, downregulation of Faim gene expression in rats fed Western diets suggested neurodegeneration.

To delineate the effects of caloric substitution with $10 \%$ of apple pomace, we compared rats fed Western/AP to rats fed Western diet using AIN-fed rats as a reference group for each analysis in order to show upregulation or downregulation of significantly expressed genes between rats fed Western/AP and rats fed Western diet (Figure 6). Results showed 15 significant differentially expressed genes. Of these, five genes had phenotypes related to neurodegeneration (i.e. Alzheimer's and Parkinson's disease): synuclein alpha, phospholipase D family, member 5, NADH-dehydrogenase ubiquinone fe-s protein 6, choline O-acetyltransferase, and frizzled class receptor 6 (Table 6).

Synuclein alpha (Snca) is a gene predominantly expressed in the brain and a biomarker of neurodegeneration in Parkinson's disease [34, 35]. Transgenic mice overexpressing Snca in the hypothalamus showed reduce body weight, adiposity, and food intake compared to normal mice [36]. In the present study, despite no significant differences in food intake, body weight, and gonadal adipose weight among diet groups, Snca gene expression was significantly upregulated in rats fed Western diet suggesting early hypothalamic dysfunction (Figure 6). Studies have reported high refined carbohydrate (e.g. sucrose and fructose) content of Western diets contributes to metabolic syndrome which indirectly promotes neurodegeneration [22, 37, 38]. Whereas, an in vitro study found polyphenols attenuated Snca aggregation and mitochondrial 
reactive oxygen species (ROS) [39]. In our study, Western diet had the highest sucrose (34\%), fructose (17\%), and the lowest polyphenol content. Snca gene expression was significantly downregulated in rats fed Western/AP diet, suggesting caloric substitution of Western diet with $10 \%$ of apple pomace attenuated early hypothalamic dysfunction. Although the Western/AP diet had the highest amount of fructose (17.45\%, and $28.4 \%$ sucrose), it also contained the highest polyphenol content. Fructose is a lipogenic substrate implicated in the pathogenesis of NAFLD [40]. Hepatic dysfunction has been reported to contribute to neurodegeneration through metabolic impairment [41]. Previously, we reported Western/AP diet attenuated Western dietinduced NAFLD [11]. Diet-induced NAFLD resulted in neurodegeneration, indicating chronic inflammation outside of the brain causes signs of Alzheimer's disease. Additionally, dietary reversal of NAFLD, reduced neuroinflammation, the activation of microglial cells, and $\beta$ amyloid plaque load indicating attenuation of NAFLD pathology plays a role in the prevention of neurodegeneration [7]. Snca has also been reported to interacts with phospholipase D, which has been implicated in neurodegeneration [42].

Phospholipase D family, member 5 (PLD5) is an integral component of neuronal cell membranes that functions by catalyzing phosphatidylcholine to phosphatidic acid and choline $[43,44,46]$. In turn, choline is required in the synthesis of acetylcholine for neurotransmission [47]. Low acetylcholine in brain regions has been implicated in energy dysregulation and neurodegenerative disease $[48,5]$. Upregulation of Snca activity downregulates PLD activity; whereas, oleic acid (18:1n-9) upregulates PLD activity both of which contribute to the modulation of choline availability $[42,45,47]$. In the present study, Snca gene expression was upregulated, brain oleic acid (18:1n-9) content decreased, and PLD5 gene expression was downregulated in rats fed Western diet suggesting phosphatidylcholine and choline deficiency in 
the hypothalamus. Studies have reported hepatic de novo synthesis of phosphatidylcholine and choline is reduced in diet-induced NAFLD due to the dysregulation of fatty acid synthesis [49]. Skinner et al. showed Western diet-induced NAFLD was attenuated by caloric substitution with $10 \%$ of apple pomace and this was accompanied by higher deposition of oleic acid (18:1n-9) in extrahepatic tissues [11, 29]. In the present study, rats fed Western/AP diet downregulated Snca gene expression, increased brain oleic acid (18:1n-9) content, and upregulated PLD5 gene expression suggesting caloric substitution of Western diet with $10 \%$ apple pomace attenuated phosphatidylcholine and choline deficiency in the hypothalamus. Western/AP diet contained the highest soluble fiber $(1.1 \%)$ content. Dietary soluble fiber has been reported to selectively retain phospholipids in the liver while excreting bile acids in the feces sustaining sufficient phosphatidylcholine levels for de novo synthesis acetylcholine in extra-hepatic tissues, such as the brain $[26,50]$. Rats fed pectin supplemented diet ( $7 \%$ by weight), a soluble fiber abundant in apples, for 4 weeks reduced phospholipids excretion while increased bile acid excretion in feces compared to rats fed a control diet (0\% by weight) [50]. Results suggest dietary soluble fibers increase the retention of phosphatidylcholine in the body, and thereby, provide a sufficient amount of choline for acetylcholine synthesis.

Choline O-acetyltransferase (Chat) is involved in the synthesis of the neurotransmitter acetylcholine by anabolizing acetyl-CoA from the mitochondrion and choline from phosphatidylcholine $[51,52]$. Chat has been implicated in the decline of memory and cognitive function in Alzheimer's disease patients through the impairment of acetylcholine synthesis [48, 53]. Chat gene expression was upregulated in rats fed Western diet suggesting compensation of impaired hypothalamic de novo acetylcholine synthesis due to the downregulation of PLD5, which provides low levels of choline for Chat synthesis of acetylcholine and thereby increase the 
risk of neurodegeneration. In contrast, Chat gene expression was downregulated in rats fed Western diet/AP compared to rats fed Western diet. Results suggest caloric substitution with $10 \%$ of apple pomace attenuated impaired hypothalamic de novo acetylcholine synthesis induced by a Western diet consumption by providing choline from PLD5 for Chat to synthesize acetylcholine and thereby, mitigates the risk of neurodegeneration. Western and Western/AP diet contained equal amounts of choline bitartrate $(3.1 \mathrm{~g} / \mathrm{kg})$; however, Western/AP diet had the highest polyphenol content. A study of age-related cognitive decline in mice maintained brainacetylcholine content provided apple juice and attributed this to the polyphenols in apple juice [54]. Devi et. al [55] reported rats fed grape seed proanthocyanidin extract increased endogenous antioxidant enzyme activity and this was accompanied by increased brain Chat activity and acetylcholine content. Mitochondria are also a source of acetyl-CoA production, which is involved in the synthesis of acetylcholine.

NADH-dehydrogenase ubiquinone fe-s protein 6 (Ndufs6) is an integral component of the mitochondrial complex I, involved in the oxidative phosphorylation pathway [56]. Deficiency of complex I is the most common mitochondrial disorder and has been linked to neurodegenerative disease $[57,58]$. Upregulated Ndufs6 gene expression in rats fed Western diet indicates Ndufs6 was required for maintaining mitochondrion integrity and function (Figure 6). Downregulated Ndufs6 gene expression in rats fed Western/AP compared to Western diet suggests caloric substitution with $10 \%$ of apple pomace attenuated mitochondrial complex I deficiency. Polyphenols have been reported to attenuate mitochondrial disorder and ROS [55, 39]. Apple pomace is a rich source of antioxidant polyphenols and Western/AP diet had the highest polyphenol content. Skinner et al. reported higher antioxidant status indicated by the highest serum total antioxidants and lowest urinary total antioxidant excretion in rats fed 
Western/AP diet compared to rats fed Western and AIN diet. Significant increase in serum antioxidants while urinary antioxidant excretion decreased suggests apple pomace increased antioxidant bioavailability [59].

Maintaining homeostasis during neurodegeneration in the CNS requires compensatory mechanisms to resolve neuronal loss mainly by neurogenesis $[60,61,62]$. Wnt/ $\beta$-catenin signaling in the brain promotes neuronal survival and neurogenesis to compensate for neuronal loss [63]. Frizzled class receptor 6 (Fzd6) is involved in the negative regulation of the Wntsignaling pathway $[64,65]$. In our study, Fzd6 gene expression was significantly downregulated in rats fed Western diet in the hypothalamus (Figure 6). Since the downregulation of Fzd6 activates the Wnt-signaling pathway, this suggests neurogenesis in the hypothalamus was active by the Wnt-signaling pathway to compensate Western diet-induced neuronal loss. Upregulation of Fzd6 gene expression in rats fed Western/AP suggests the Wnt-signaling pathway is inactive due to neurodegeneration was attenuated by caloric substitution with $10 \%$ of apple pomace in Western diet.

Figure 7 shows a hypothetical illustration of the pathogenesis and attenuation of hypothalamic dysfunction by caloric substitution with $10 \%$ of apple pomace in the Western diet. Early indicators of neurodegeneration in rats consuming a Western diet were ameliorated by caloric substitution with $10 \%$ apple pomace. Apple pomace mitigated neurodegeneration by 1) attenuating phosphatidylcholine and choline deficiency through the attenuation of NAFLD, 2) possible fatty acid transport from the liver increasing oleic acid (18:1n-9) concentration in the brain, and 3) regulating gene expression Snca, PLD5, Chat, Ndufs6, and Fzd6 implicated in de novo acetylcholine synthesis and neurogenesis in the hypothalamus. These health-promoting 
effects on brain lipid profile and gene expression were attributed to the high soluble fiber and polyphenol content of apple pomace.

In conclusion, based on the current exploration of gene expression, apple pomace provides insight into mechanisms of action and pre-clinical evidence as a potential therapy to attenuate the pathogenesis of Western diet-induced hypothalamic dysfunction implicated in neurodegenerative diseases. Caloric substitution of Western diet with $10 \%$ of apple pomace significantly altered fatty acid composition in the brain, and attenuated expression of genes related to hypothalamic dysfunction and neurodegeneration in growing female rats. Investigating the potential of apple pomace for human consumption can decrease health care costs for treating neurodegenerative diseases as well as offers a solution for reducing environmental pollution and the expense of waste disposal. Therefore, apple pomace merits further study as a possible sustainable functional food. 


\section{References}

1- Varela L, Horvath TL. Leptin and Insulin Pathways in POMC and AgRP Neurons that Modulate Energy Balance and Glucose Homeostasis. EMBO reports. 2012;13:1079-1086.

2- Cai D, Liu T. Hypothalamic Inflammation: A Double-Edged Sword to Nutritional Diseases. Annals of the New York Academy of Sciences. 2011;1243:E1-E39.

3- Vercruysse P, Vieau D, Blum D, et al. Hypothalamic Alterations in Neurodegenerative Diseases and Their Relation to Abnormal Energy Metabolism. Frontiers in Molecular Neuroscience. 2018;11:2.

4- Hampel H, Mesulam M-M, Cuello AC, et al. The Cholinergic System in the Pathophysiology and Treatment of Alzheimer's Disease. Brain. 2018;141:1917-1933.

5- Jeong JH, Lee DK, Jo Y-H. Cholinergic Neurons in the Dorsomedial Hypothalamus Regulate Food Intake. Molecular Metabolism. 2017;6:306-312.

6- Sherriff JL, O’Sullivan TA, Properzi C, et al. Choline, Its Potential Role in Nonalcoholic Fatty Liver Disease, and the Case for Human and Bacterial Genes. Advances in Nutrition. 2016;7:5-13.

7- Kim DG, Krenz A, Toussaint LE, et al. Non-Alcoholic Fatty Liver Disease Induces Signs of Alzheimer's Disease (AD) in Wild-Type Mice and Accelerates Pathological Signs of AD in an AD Model. Journal of Neuroinflammation. 2016;13:1.

8- Skinner RC, Gigliotti JC, Ku K-M, et al. A Comprehensive Analysis of the Composition, Health Benefits, and Safety of Apple Pomace. Nutrition Reviews. 2018;76:893-909.

9- Valdearcos M, Robblee MM, Benjamin DI, et al. Microglia Dictate the Impact of Saturated Fat Consumption on Hypothalamic Inflammation and Neuronal Function. Cell Reports. 2014;9:2124-2138.

10- (2020). 2020 Alzheimer's Disease Facts and Figures. Alzheimer's \& Dementia. 2020;16:391-460.

11-Skinner RC, Warren DC, Lateef SN, et al. Apple Pomace Consumption Favorably Alters Hepatic Lipid Metabolism in Young Female Sprague-Dawley Rats Fed a Western Diet. Nutrients. 2018;10:1882.

12- Conrad Z, Niles MT, Neher DA, et al. Relationship Between Food Waste, Diet Quality, and Environmental Sustainability. PLOS ONE. 2018;13:e0195405.

13- Blainski A, Lopes GC, de Mello JCP. Application and Analysis of the Folin Ciocalteu Method for the Determination of the Total Phenolic Content from Limonium Brasiliense L. Molecules. 2013;18:6852-6865. 
14- Bligh EG, Dyer WJ. A Rapid Method of Total Lipid Extraction and Purification. Canadian Journal of Biochemistry and Physiology. 1959;37:911-917.

15- Fritsche KL, Johnston PV. Effect of Dietary $\alpha$-Linolenic Acid on Growth, Metastasis, Fatty Acid Profile and Prostaglandin Production of Two Murine Mammary Adenocarcinomas. The Journal of Nutrition. 1990;120:1601-1609.

16- Rumple A, McMurray M, Johns J, et al. 3-Dimensional Diffusion Tensor Imaging (DTI) Atlas of the Rat Brain. PLOS ONE. 2013;8:e67334.

17- Peri S, Roberts S, Kreko IR, et al. Read Mapping and Transcript Assembly: A Scalable and High-Throughput Workflow for the Processing and Analysis of Ribonucleic Acid Sequencing Data. Frontiers in Genetics. 2020;10:1361.

18- Gibbs RA, Weinstock GM, Metzker ML, et al. Genome Sequence of the Brown Norway Rat Yields Insights Into Mammalian Evolution. Nature. 2004;428:493-521.

19- Marini F, Binder H. pcaExplorer: An R/Bioconductor Package for Interacting with RNA-seq Principal Components. BMC Bioinformatics. 2019;20:331.

20- Hunt SE, McLaren W, Gil L, et al. Ensembl Variation Resources. Database [Internet]. 2018 [cited 2020 Oct 21];2018. Available from: https://doi.org/10.1093/database/bay119.

21- Mi H, Muruganujan A, Huang X, et al. Protocol Update for Large-Scale Genome and Gene Function Analysis with the PANTHER Classification System (v.14.0). Nature Protocols. 2019;14:703-721.

22- Gimenez da Silva-Santi L, Masetto Antunes M, Mori MA, et al. Brain Fatty Acid Composition and Inflammation in Mice Fed with High-Carbohydrate Diet or High-Fat Diet. Nutrients. 2018;10:1277.

23- Valdearcos M, Robblee MM, Benjamin DI, et al. Microglia Dictate the Impact of Saturated Fat Consumption on Hypothalamic Inflammation and Neuronal Function. Cell Reports. 2014;9:2124-2138.

24- Taha AY. Linoleic Acid Good or Bad for the Brain? NPJ Sci Food. 2020;4:1-1.

25- Taha AY, Blanchard HC, Cheon Y, et al. Dietary Linoleic Acid Lowering Reduces Lipopolysaccharide-Induced Increase in Brain Arachidonic Acid Metabolism. Molecular Neurobiology. 2017;54:4303-4315.

26- Kritchevskya D, Tepper SA, Satchithanandam S, et al. Dietary Fiber Supplements: Effects on Serum and Liver Lipids and on Liver Phospholipid Composition in Rats. Lipids. 1988;23:318-321.

27-Song J, Kim Y-S, Lee DH, et al. Neuroprotective Effects of Oleic Acid in Rodent Models of Cerebral Ischaemia. Scientific Reports. 2019;9:10732. 
28- Mesa-Herrera F, Taoro-González L, Valdés-Baizabal C, et al. Lipid and Lipid Raft Alteration in Aging and Neurodegenerative Diseases: A Window for the Development of New Biomarkers. Int J Mol Sci. 2019;20:3810.

29- Skinner RC, Warren DC, Agarwal G, et al. Apple Pomace Influences Liver-Adipose Tissue Inflammatory Crosstalk in Young Female Rats Consuming a Western Diet. The FASEB Journal. 2019;33:872.3-872.3.

30- Hussain G, Schmitt F, Loeffler J-P, et al. Fatting the Brain: A Brief of Recent Research. Frontiers in Cellular Neuroscience. 2013;7:144.

31- Coiled-coil domain-containing protein 72-like, RGD ID 6497475 Rat Genome Database Web Site, Medical College of Wisconsin, Milwaukee, Wisconsin. World Wide Web (URL: http://rgd.mcw.edu/rgdweb/report/gene/main.html?id=6497475). (Aug, 2020).

32- Fas apoptotic inhibitory molecule, RGD ID 620572, Rat Genome Database Web Site, Medical College of Wisconsin, Milwaukee, Wisconsin. World Wide Web (URL: http://rgd.mcw.edu/rgdweb/report/gene/main.html?id=620572). (Aug, 2020).

33- Moubarak RS, Planells-Ferrer L, Urresti J, et al. FAIM-L Is an IAP-Binding Protein That Inhibits XIAP Ubiquitinylation and Protects from Fas-Induced Apoptosis. Journal of Neuroscience. 2013;33:19262-19275.

34- Synuclein alpha, RGD ID 3729, Rat Genome Database Web Site, Medical College of Wisconsin, Milwaukee, Wisconsin. World Wide Web (URL: http://rgd.mcw.edu/rgdweb/report/gene/main.html?id=3729). (Aug, 2020).

35- Stefanis L. $\alpha$-Synuclein in Parkinson's Disease. Cold Spring Harbor Perspectives in Medicine. 2012;2.

36- Cuvelier E, Méquinion M, Leghay C, et al. Overexpression of Wild-Type Human AlphaSynuclein Causes Metabolism Abnormalities in Thy1-aSYN Transgenic Mice. Frontiers in Molecular Neuroscience. 2018;11:321.

37- da Silva-Santi LG, Antunes MM, Caparroz-Assef SM, et al. Liver Fatty Acid Composition and Inflammation in Mice Fed with High-Carbohydrate Diet or High-Fat Diet. Nutrients. 2016;8:682

38- Harrell CS, Zainaldin C, McFarlane D, et al. High-Fructose Diet During Adolescent Development Increases Neuroinflammation and Depressive-Like Behavior Without Exacerbating Outcomes After Stroke. Brain, Behavior, and Immunity. 2018;73:340-351.

39- Macedo D, Jardim C, Figueira I, et al. (Poly)phenol-Digested Metabolites Modulate AlphaSynuclein Toxicity by Regulating Proteostasis. Scientific Reports. 2018;8:6965. 
40- Mock K, Lateef S, Benedito VA, et al. High-Fructose Corn Syrup-55 Consumption Alters Hepatic Lipid Metabolism and Promotes Triglyceride Accumulation. The Journal of Nutritional Biochemistry. 2017;39:32-39.

41- Estrada LD, Ahumada P, Cabrera D, et al. Liver Dysfunction as a Novel Player in Alzheimer's Progression: Looking Outside the Brain. Frontiers in Aging Neuroscience. 2019;11:174.

42- Conde MA, Alza NP, Iglesias González PA, et al. Phospholipase D1 Downregulation by $\alpha-$ Synuclein: Implications for Neurodegeneration in Parkinson's Disease. Biochimica et Biophysica Acta (BBA) - Molecular and Cell Biology of Lipids. 2018;1863:639-650.

43- Phospholipase D family, member 5, RGD ID 1562609, Rat Genome Database Web Site, Medical College of Wisconsin, Milwaukee, Wisconsin. World Wide Web (URL: http://rgd.mcw.edu/rgdweb/report/gene/main.html?id=1562609). (Aug, 2020).

44- Oliveira TG, Di Paolo G. Phospholipase D in Brain Function and Alzheimer's Disease. Biochim Biophys Acta. 2010/04/23 ed. 2010;1801:799-805.

45- Mendez-Gomez HR, Singh J, Meyers C, et al. The Lipase Activity of Phospholipase D2 is Responsible for Nigral Neurodegeneration in a Rat Model of Parkinson's Disease. Neuroscience. 2018/03/09 ed. 2018;377:174-183.

46- Park K, Kim J, Nagashima Y, et al. Detection of Choline and Phosphatidic Acid (PA) Catalyzed by Phospholipase D (PLD) using MALDI-QIT-TOF/MS with 9-aminoacridine matrix. null. 2014;78:981-988.

47- Hattori H, Kanfer JN. Synaptosomal Phospholipase D: Potential Role in Providing Choline for Acetylcholine Synthesis. Biochemical and Biophysical Research Communications. 1984;124:945-949.

48- Maurer SV, Williams CL. The Cholinergic System Modulates Memory and Hippocampal Plasticity via Its Interactions with Non-Neuronal Cells. Frontiers in Immunology. 2017;8:1489.

49- Van der Veen JN, Kennelly JP, Wan S, et al. The Critical Role of Phosphatidylcholine and Phosphatidylethanolamine Metabolism in Health and Disease. Biochimica et Biophysica Acta (BBA) - Biomembranes. 2017;1859:1558-1572.

50- Garcia-Diez F, Garcia-Mediavilla V, Bayon JE, et al. Pectin Feeding Influences Fecal Bile Acid Excretion, Hepatic Bile Acid and Cholesterol Synthesis and Serum Cholesterol in Rats. The Journal of Nutrition. 1996;126:1766-1771.

51- Choline O-acetyltransferase, RGD ID 1304627, Rat Genome Database Web Site, Medical College of Wisconsin, Milwaukee, Wisconsin. World Wide Web (URL:

http://rgd.mcw.edu/rgdweb/report/gene/main.html?id=1304627). (Aug, 2020). 
52- Park D, Joo SS, Kim TK, et al. Human Neural Stem Cells Overexpressing Choline Acetyltransferase Restore Cognitive Function of Kainic Acid-Induced Learning and Memory Deficit Animals. Cell Transplant. 2012;21:365-371.

53- Mubumbila V, Sutter A, Ptok U, et al. Identification of a Single Nucleotide Polymorphism in the Choline Acetyltransferase gene Associated with Alzheimer's Disease. Neuroscience Letters. 2002;333:9-12.

54-Chan A, Graves V, Shea TB. Apple Juice Concentrate Maintains Acetylcholine Levels Following Dietary Compromise. Journal of Alzheimer's Disease. 2006;9:287-291.

55- Devi A, Jolitha AB, Ishii N. Grape Seed Proanthocyanidin Extract (GSPE) and Antioxidant Defense in the Brain of Adult Rats. Medical science monitor : international medical journal of experimental and clinical research. 2006;12:BR124-9.

56- NADH:ubiquinone oxidoreductase subunit S6, RGD ID 3156, Rat Genome Database Web Site, Medical College of Wisconsin, Milwaukee, Wisconsin. World Wide Web (URL: http://rgd.mcw.edu/rgdweb/report/gene/main.html?id=3156). (Aug, 2020).

57- Forbes JM, Ke B-X, Nguyen T-V, et al. Deficiency in Mitochondrial Complex I Activity Due to Ndufs6 Gene Trap Insertion Induces Renal Disease. Antioxidants \& Redox Signaling. 2013;19:331-343.

58-Sharma LK, Lu J, Bai Y. Mitochondrial Respiratory Complex I: Structure, Function and Implication in Human Diseases. Curr Med Chem. 2009;16:1266-1277.

59- Skinner RC, Warren DC, Naveed M, et al. Apple Pomace Improves Liver and Adipose Inflammatory and Antioxidant Status in Young Female Rats Consuming a Western Diet. Journal of Functional Foods. 2019;61:103471.

60- Kuhn HG, Palmer TD, Fuchs E. Adult Neurogenesis: A Compensatory Mechanism for Neuronal Damage. European Archives of Psychiatry and Clinical Neuroscience. 2001;251:152-158.

61- Moreno-Jiménez EP, Flor-García M, Terreros-Roncal J, et al. Adult Hippocampal Neurogenesis is Abundant in Neurologically Healthy Subjects and Drops Sharply in Patients with Alzheimer's Disease. Nature Medicine. 2019;25:554-560.

62- Pierce AA, Xu AW. De Novo Neurogenesis in Adult Hypothalamus as a Compensatory Mechanism to Regulate Energy Balance. Journal of Neuroscience. 2010;30:723-730.

63- Jia L, Piña-Crespo J, Li Y. Restoring Wnt/ $\beta$-catenin Signaling is a Promising Therapeutic Strategy for Alzheimer's Disease. Molecular Brain. 2019;12:104. 
64- Frizzled class receptor 6, RGD ID 628816, Rat Genome Database Web Site, Medical College of Wisconsin, Milwaukee, Wisconsin. World Wide Web (URL:

http://rgd.mcw.edu/rgdweb/report/gene/main.html?id=628816). (Aug, 2020).

65-Golan T, Yaniv A, Bafico A, et al. The Human Frizzled 6 (HFz6) Acts as a Negative Regulator of the Canonical Wnt $\beta$-Catenin Signaling Cascade. Journal of Biological Chemistry. 2004;279:14879-14888. 
Table 1. Nutrient composition of experimental diets fed to growing female rats.

\begin{tabular}{|c|c|c|c|c|}
\hline & & Diets $^{1}$ & & \\
\hline & AIN & Western & Western/AP & Apple Pomace \\
\hline${\text { Ingredients }(\mathrm{g} / \mathrm{kg})^{1}}^{1}$ & & & & (g/100g) \\
\hline Apple pomace & 0.0 & 0.0 & 100.0 & 100 \\
\hline Corn Starch & 397.486 & 63.36 & 57.96 & - \\
\hline Maltodextrin & 132.0 & 60.0 & 60.0 & - \\
\hline Sucrose & 100.0 & 340.0 & 283.9 & 13.9 \\
\hline Fructose & 50 & 170 & 174.45 & 33.5 \\
\hline Total Dietary Fiber & 50.0 & 50.0 & 50.0 & 33.2 \\
\hline Insoluble Fiber $^{2}$ & 50.0 & 50.0 & 39.0 & 22.2 \\
\hline Soluble Fiber ${ }^{3}$ & 0.0 & 0.0 & 11.0 & 11.0 \\
\hline Anhydrous Milkfat & 0.0 & 210.0 & 210.0 & - \\
\hline Soybean Oil & 70.0 & 20.0 & 18.7 & - \\
\hline Casein & 200.0 & 195.0 & 191.0 & - \\
\hline L-Cystine & 3.0 & 3.0 & 3.0 & - \\
\hline Vitamin Mix & 10.0 & 12.5 & 12.5 & - \\
\hline Mineral Mix & 35.0 & 43.0 & 43.0 & - \\
\hline Choline Bitatrate & 2.5 & 3.1 & 3.1 & - \\
\hline TBHQ antioxidant & 0.014 & 0.04 & 0.04 & - \\
\hline Polyphenols & 0.0015 & 0.0008 & 0.0032 & 0.29 \\
\hline Macronutrients (\% & & & & \\
\hline Protein & 18.8 & 14.8 & 14.8 & 3.6 \\
\hline Fat & 17.2 & 44.6 & 44.8 & 1.3 \\
\hline Carbohydrate & 63.9 & 40.6 & 40.4 & 68.1 \\
\hline Calories (kcal/g) & 3.8 & 4.7 & 4.7 & 3.9 \\
\hline
\end{tabular}

${ }^{1}$ Abbreviations: AIN, the American Institute of Nutrition; AP, apple pomace; TBHQ, tertbutylhydroquinone. ${ }^{2}$ Insoluble fiber is cellulose. ${ }^{3}$ Soluble fiber is mainly pectin [1]. 
Table 2. Fatty acid analysis of experimental rodent diets.

\begin{tabular}{lccc}
\hline $\begin{array}{l}\text { Fatty Acids } \\
\text { Measurements (mg/g) }\end{array}$ & AIN & $\begin{array}{c}\text { Diets } \\
\text { Western }\end{array}$ & Western/AP \\
\hline SFAs & & & \\
Palmitic acid (16:0) & $113.6 \pm 0.9^{\mathrm{c}}$ & $321.9 \pm 0.3^{\mathrm{b}}$ & $329.2 \pm 3.0^{\mathrm{a}}$ \\
Stearic acid (18:0) & $35.6 \pm 2.5^{\mathrm{b}}$ & $99.4 \pm 1.1^{\mathrm{a}}$ & $102.4 \pm 0.6^{\mathrm{a}}$ \\
\hline MUFAs & & & \\
Palmitoleic acid (16:1n-7) & $0 \pm 0.00^{\mathrm{b}}$ & $14.4 \pm 0.01^{\mathrm{a}}$ & $14.4 \pm 0.02^{\mathrm{a}}$ \\
Oleic acid (18:1n-9) & $190.9 \pm 1.0^{\mathrm{b}}$ & $229.6 \pm 1.1^{\mathrm{a}}$ & $229.5 \pm 1.7^{\mathrm{a}}$
\end{tabular}

\section{PUFAs}

\section{Omega-6}
Linoleic acid (18:2n-6)
$501.2 \pm 5.5^{\mathrm{a}}$
$69.9 \pm 0.9^{\mathrm{b}}$
$70.4 \pm 0.6^{b}$
Arachidonic acid (20:4n-6)

$$
0 \pm 0.00^{\mathrm{b}}
$$
$1.3 \pm 0.00^{\text {a }}$
$1.4 \pm 0.00^{\text {a }}$

\section{Omega-3}
$\alpha$-linolenic acid (18:3n-3)

$$
\begin{gathered}
70.8 \pm 0.13^{a} \\
0 \pm 0.00 \\
0 \pm 0.00
\end{gathered}
$$$$
10.4 \pm 0.1^{\mathrm{b}}
$$$$
10.5 \pm 0.2^{b}
$$
EPA (20:5n-3)
$0 \pm 0.00$
$0 \pm 0.00$
DHA (22:6n-3)
$0 \pm 0.00$
$0 \pm 0.00$

\footnotetext{
${ }^{1}$ Values expressed as mean \pm standard error of the mean (SEM, $n=5$ samples/group).

Different superscript letters a, b, and $\mathrm{c}$ within. The same row indicates significant difference at $p<0.05$ by one-way ANOVA followed by Tukey's test. Abbreviations: DHA, docosahexaenoic acid; EPA, eicosapentaenoic acid; SFAs, saturated fatty acids; MUFAs, monounsaturated fatty acids; PUFAs, polyunsaturated fatty.
} 
Table 3. Effect of consumption of different diets on total caloric intake, body weight, and brain weight for 8 weeks.

\begin{tabular}{|c|c|c|c|c|}
\hline \multirow[b]{2}{*}{ Measurements } & \multicolumn{3}{|c|}{ Diets $^{1}$} & \multirow[b]{2}{*}{ p-Value } \\
\hline & AIN & Western & Western/AP & \\
\hline Caloric intake (kcal) & $2768 \pm 85^{b}$ & $3335 \pm 71^{\mathrm{a}}$ & $3336 \pm 134^{\mathrm{a}}$ & $<0.0006$ \\
\hline Lipid intake (kcal) & $476 \pm 15^{b}$ & $1487 \pm 32^{a}$ & $1494 \pm 60^{a}$ & $<0.0001$ \\
\hline CHO intake (kcal) & $1769 \pm 54^{\mathrm{a}}$ & $1354 \pm 29^{b}$ & $1347 \pm 54^{b}$ & $<0.0001$ \\
\hline Protein intake (kcal) & $520 \pm 16$ & $494 \pm 10$ & $494 \pm 20$ & 0.4022 \\
\hline Feed Efficiency Ratio (\%) & $17 \pm 0.01$ & $18 \pm 0.01$ & $18 \pm 0.01$ & 0.13 \\
\hline Initial bwt (g) & $95 \pm 2.5$ & $95 \pm 2.4$ & $95 \pm 2.5$ & 0.99 \\
\hline Final bwt (g) & $216 \pm 4^{b}$ & $228 \pm 5^{a b}$ & $234 \pm 5^{\mathrm{a}}$ & $<0.0373$ \\
\hline Gonadal fat pad weight (g) & $4.11 \pm 0.26^{b}$ & $5.43 \pm 0.50^{a}$ & $5.96 \pm 0.23^{a}$ & $<0.0022$ \\
\hline Relative brain weight (mg/bwt) & $7.13 \pm 0.17$ & $7.02 \pm 0.12$ & $6.80 \pm 0.14$ & 0.28 \\
\hline Absolute brain weight (g) & $1.54 \pm 0.03$ & $1.59 \pm 0.02$ & $1.60 \pm 0.03$ & 0.22 \\
\hline
\end{tabular}

\footnotetext{
${ }^{1}$ Values expressed as mean \pm SEM ( $n=6-8$ rats/group). Different superscript letters a and $b$ within the same row. Indicate significant difference at $p<0.05$ by one-way ANOVA followed by Tukey's test. Abbreviations: Bwt, body weight; $\mathrm{CHO}$, carbohydrate.
} 
Table 4. Lipid content and Fatty acid analysis of brain of rats fed different experimental diets.

\begin{tabular}{lcccc}
\hline Lipid/Fatty Acids (\%) & AIN & Western & Western/AP & p-Value \\
\hline Total lipid content & $7.21 \pm 0.28^{\mathrm{b}}$ & $7.4 \pm 0.022^{\mathrm{a}}$ & $6.58 \pm 0.43^{\mathrm{b}}$ & $<0.0001$ \\
\hline SFAs & & & & \\
Palmitic acid (16:0) & $22.01 \pm 0.85$ & $19.78 \pm 1.36$ & $22.49 \pm 1.67$ & 0.32 \\
Stearic acid (18:0) & $20.35 \pm 0.85$ & $17.71 \pm 1.03$ & $17.65 \pm 0.81$ & 0.07 \\
\hline MUFAs & & & & \\
Palmitoleic acid (16:1n-7) & N/A & N/A & N/A & \\
Oleic acid (18:1n-9) & $5.41 \pm 0.28^{\mathrm{b}}$ & $5.15 \pm 0.19^{\mathrm{b}}$ & $7.51 \pm 0.54^{\mathrm{a}}$ & $<0.0005$ \\
\hline PUFAs & & & &
\end{tabular}

\section{Omega-6}

Linoleic acid (18:2n-6)

Arachidonic acid (20:4n-6)

$$
4.37 \pm 0.47^{\mathrm{a}}
$$

N/A

N/A

N/A

$$
2.93 \pm 0.49^{\mathrm{ab}}
$$

$2.75 \pm 0.27^{\mathrm{b}}$

N/A

N/A

\section{Omega-3}

$\alpha$-linolenic acid (18:3n-3)

EPA (20:5n-3)

DHA (22:6n-3)

$9.70 \pm 1.32$

N/A

N/A

N/A

N/A

Brain lipid content in $\%$, and relative $\%$ brain total fatty acid profile of growing rats consuming experimental diets following eight weeks of feeding. ${ }^{1}$ Values expressed as mean \pm standard error of the mean (SEM, $n=6-8$ samples/group). Different superscript letters a, b, and c within. The same row indicates significant difference at $p<0.05$ by one-way ANOVA followed by Tukey's test. Abbreviations: DHA, docosahexaenoic acid; EPA, eicosapentaenoic acid; SFAs, saturated fatty acids; MUFAs, monounsaturated fatty acids; PUFAs, polyunsaturated fatty. 
Table 5. Differential gene expression analysis of hypothalamus rodents fed diets substituted with apple pomace $(10 \% \mathrm{~g} / \mathrm{kg})$.

\begin{tabular}{lcccc}
\hline & \multicolumn{4}{c}{ Analyses } \\
Pipeline's & Gene & AIN vs & WE/AP vs & q-Value \\
Significant gene name & Symbol & (WE-WE/AP) & WE & \\
\hline
\end{tabular}

\section{VICE DESeq2}

Fas apoptotic inhibitory molecule

\begin{tabular}{|c|c|c|c|}
\hline Faim & $-4.812^{*}$ & 1.002 & $<0.005$ \\
\hline LOC100910678 & $1.654 *$ & 0.881 & $<0.0005$ \\
\hline Vwa5a & 1.941 & $-5.569^{*}$ & $<0.047$ \\
\hline Ndufs6 & 3.102 & $-4.302 *$ & $<0.05$ \\
\hline Inpp5f & -0.179 & $0.340 *$ & $<0.05$ \\
\hline Snca & 0.348 & $-0.546^{*}$ & $<0.05$ \\
\hline Cent1 & N/A & $-1.535^{*}$ & $<0.05$ \\
\hline Rffl & N/A & $0.438^{*}$ & $<0.05$ \\
\hline Pld5 & N/A & $-0.622 *$ & $<0.06$ \\
\hline Chat & N/A & $-1.383^{*}$ & $<0.05$ \\
\hline Fzd6 & -0.324 & $0.848 *$ & $<0.05$ \\
\hline Mir2964 & -1.257 & $2.310 *$ & $<0.05$ \\
\hline Nbl1 & 0.143 & $0.256^{*}$ & $<0.06$ \\
\hline Slc35f3 & N/A & $-0.459 *$ & $<0.066$ \\
\hline LOC100912538 & -1.318 & $-3.179 *$ & $<0.07$ \\
\hline Igfbp4 & N/A & $0.951 *$ & $<0.07$ \\
\hline Hs3st1 & N/A & $-0.440 *$ & $<0.098$ \\
\hline
\end{tabular}

Coiled-coil domain-containing protein 72-like

Von Willebrand factor A domain containing $5 \mathrm{~A}$

NADH dehydrogenase (ubiquinone)

Fe-S protein 6

Inositol polyphosphate-5-

phosphatase $\mathrm{F}$

Synuclein alpha

Cyclin T1

RING finger FYVE-like ligase

Phospholipase D family, member 5

Choline O-acetyltransferase

Frizzled class receptor 6

MicroRNA 2964

NBL1, DAN family BMP

antagonist

Solute carrier family 35 , member

F3

Centrin-3-like

Insulin-like growth factor binding protein 4

Heparan sulfate-glucosamine 3-

sulfotransferase 1 
Table 6. Phenotype, disease and trait of significantly expressed genes hypothalamus of rats fed Western/AP compared to Western diet.

\begin{tabular}{|c|c|c|}
\hline Gene Symbol & Phenotype & Gene ID \\
\hline Vwa5a & Schizophrenia, Dwarfism & ENSRNOG00000046414 \\
\hline Ndufs6 & $\begin{array}{c}\text { Mitochondrial complex I } \\
\text { deficiency, Alzheimer's and } \\
\text { Parkinson's disease pathway }\end{array}$ & ENSRNOG00000049394 \\
\hline Inpp5f & N/A & ENSRNOG00000020388 \\
\hline Snca $^{1}$ & $\begin{array}{c}\text { Alzheimer's Disease, Brain } \\
\text { Injuries, Cocaine-Related } \\
\text { Disorders, Creutzfeldt-Jakob } \\
\text { disease, GM2 gangliosidosis, } \\
\text { Lewy body dementia, } \\
\text { Parkinson's disease }{ }^{1}, \\
\text { Parkinsonian Disorders, Pick's } \\
\text { disease, Amphetamine abuse, } \\
\text { Bipolar Disorder, Mental } \\
\text { depression, Multiple System } \\
\text { Atrophy, Myeloid leukemia, } \\
\text { Neurilemmoma, Pantothenate } \\
\text { Kinase-Associated } \\
\text { Neurodegeneration, } \\
\text { Schizophrenia, Vascular } \\
\text { dementia. }\end{array}$ & ENSRNOG00000008656 \\
\hline Ccnt1 & $\begin{array}{c}\text { Diabetes Mellitus Experimental, } \\
\text { Congestive heart failure }\end{array}$ & ENSRNOG00000053054 \\
\hline Rffl & Hypertension & ENSRNOG00000007596 \\
\hline Pld5 & $\begin{array}{c}\text { Neurodevelopmental Disorders, } \\
\text { Fumarase deficiency, autistic } \\
\text { disorder, Hereditary } \\
\text { Leiomyomatosis and Renal Cell } \\
\text { Cancer, Senior-Loken } \\
\text { Syndrome } 7 \text { Megalencephaly- } \\
\text { Polymicrogyria-Polydactyly- } \\
\text { Hydrocephalus Syndrome } 2\end{array}$ & ENSRNOG00000003997 \\
\hline Chat & $\begin{array}{c}\text { Alzheimer's Disease, } \\
\text { Huntington's Disease, } \\
\text { Asthma, congenital myasthenic } \\
\text { syndrome } 6\end{array}$ & ENSRNOG00000025012 \\
\hline
\end{tabular}




\begin{tabular}{lcc}
\hline Fzd6 $^{\mathbf{1}}$ & $\begin{array}{c}\text { Alzheimer disease-presenilin } \\
\text { pathway }\end{array}$ & ENSRNOG00000004660 \\
\hline Mir2964 & N/A & ENSRNOG00000035452 \\
\hline Nbl1 & N/A & ENSRNOG00000049402 \\
\hline Slc35f3 & N/A & ENSRNOG00000049456 \\
\hline LOC100912538 & N/A & ENSRNOG00000050877 \\
\hline Igfbp4 & N/A & ENSRNOG00000010635 \\
\hline Hs3st1 & Coronary artery disease & ENSRNOG00000010598 \\
\hline
\end{tabular}

Phenotype data was obtained from the BioMart Ensemble database with viable link to Rat Genome Data (Aug. 2020). N/A, Not Available. ${ }^{1}$ Fzd6 and Snca pathway data were obtained from Panther gene analysis tool 


\section{Figure Legends}

Figure 1. An overview flowchart of RNA-Seq experiment and data analysis

Figure 2. Global Principal Component Analysis biplot of hypothalamic tissues from rats fed different diets. Abbreviations AIN, the American Institute of Nutrition; WE, Western; WE/AP, Western/AP.

Figure 3. Global hierarchical clustering analyses of total gene expression $(32,883$ genes) in the hypothalamus rats fed different diet. Values expressed in shifted logarithm transformation normalized counts performed using VICE DEseq2. The darker the color shade the more gene expression. Each line is considered a gene in each hypothalamus of rat sample fed different diet . Abbreviations AIN, the American Institute of Nutrition; WE, Western; WE/AP, Western/AP.

Figure 4. Hierarchical clustering analysis of gene expression related to food-intake pathway of hypothalamus rats fed different diet that are not significant (11 genes). The darker the color shade, the more gene expression. Abbreviations AIN, the American Institute of Nutrition; WE, Western; WE/AP, Western/AP; Cart, Cocaine- and amphetamine-regulated transcript; STAT3, signal transducer and activator of transcription 3; POMC, Pro-opiomelanocortin; InsR, Insulin receptors; FOXO1, Forkhead box protein O1; Jak3, Janus kinase 3; Pi3k, Phosphoinositide 3kinases; Mc4r, Melanocortin 4 receptor; AgRP, Agouti-related protein neurons; LepR, Leptin receptors; Npy, Neuropeptide Y.

Figure 5. Hierarchical clustering analysis of significant gene expression ( $q<0.10,15$ genes) of hypothalamus rats fed Western vs Western/AP. Values are expressed in shifted logarithm transformation normalized counts performed using VICE DESeq2. The darker the color shade, the more gene expression. Abbreviations AIN, the American Institute of Nutrition; WE, Western; WE/AP, Western/AP; Inpp5f, Inositol polyphosphate-5-phosphatase F; Nbl1, NBL1, DAN family BMP antagonist; Snca, Synuclein alpha; Rffl, Ring finger FYVE-like ligase; Hs3st1, Heparan sulfate-glucosamine 3-sulfotransferase 1; Slc35f3, Solute carrier family 35 member F3; Igfbp4, Insulin-like growth factor binding protein 4; Pld5, Phospholipase D family, member 5; Ndufs6, NADH dehydrogenase (ubiquinone) Fe-S protein 6; Chat, Choline Oacetyltransferase; Fzd6, Frizzled class receptor 6; LOC100912538, Centrin-3-like; Ccnt1, Cyclin T1; Mir2964, MicroRNA 2964; Vwa5a, Von Willebrand factor A domain containing 5A.

Figure 6. Upregulated and downregulated of differentially expressed genes $(\mathrm{q}<0.10) *$ of hypothalamus rats fed Western (AINvsWE) compared to Western/AP (AINvsWE/AP) diet. Values are expressed in $\log 2$ foldchange with standard error. Abbreviations AIN, the American Institute of Nutrition; WE, Western; WE/AP, Western/AP.

Figure 7. Hypothetical illustration shows effects of Western diet and Western/Apple pomace on brain lipid profile and hypothalamic neurons in rats. Abbreviations: NAFLD, Non-alcoholic fatty liver disease; Pld5, Phospholipase D family, member 5; Snca, Synuclein alpha; Ndufs6, NADH dehydrogenase (ubiquinone) Fe-S protein 6; Chat, Choline O-acetyltransferase; Fzd6, Frizzled class receptor 6 
Figure 1.

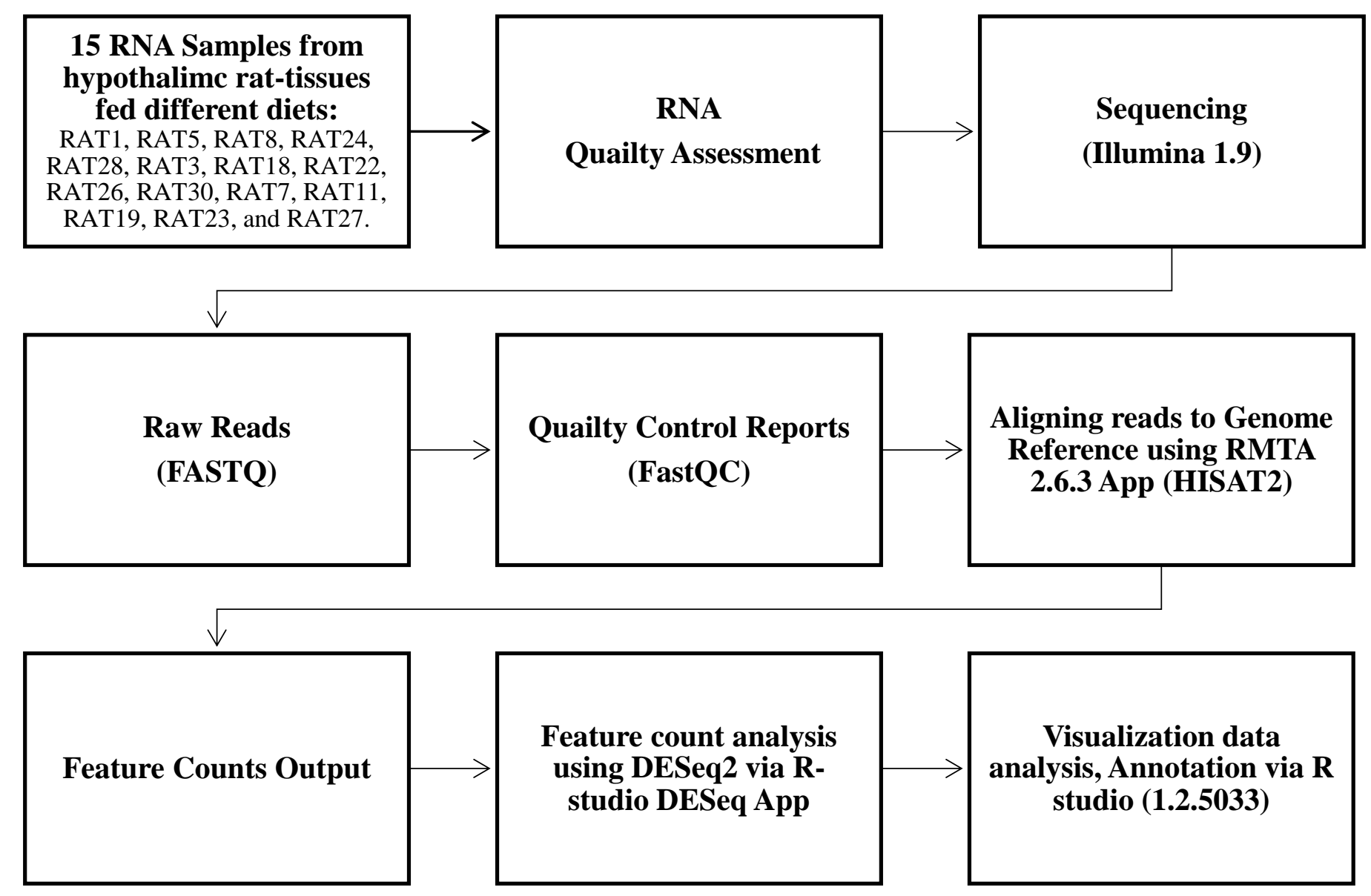


Figure 2.

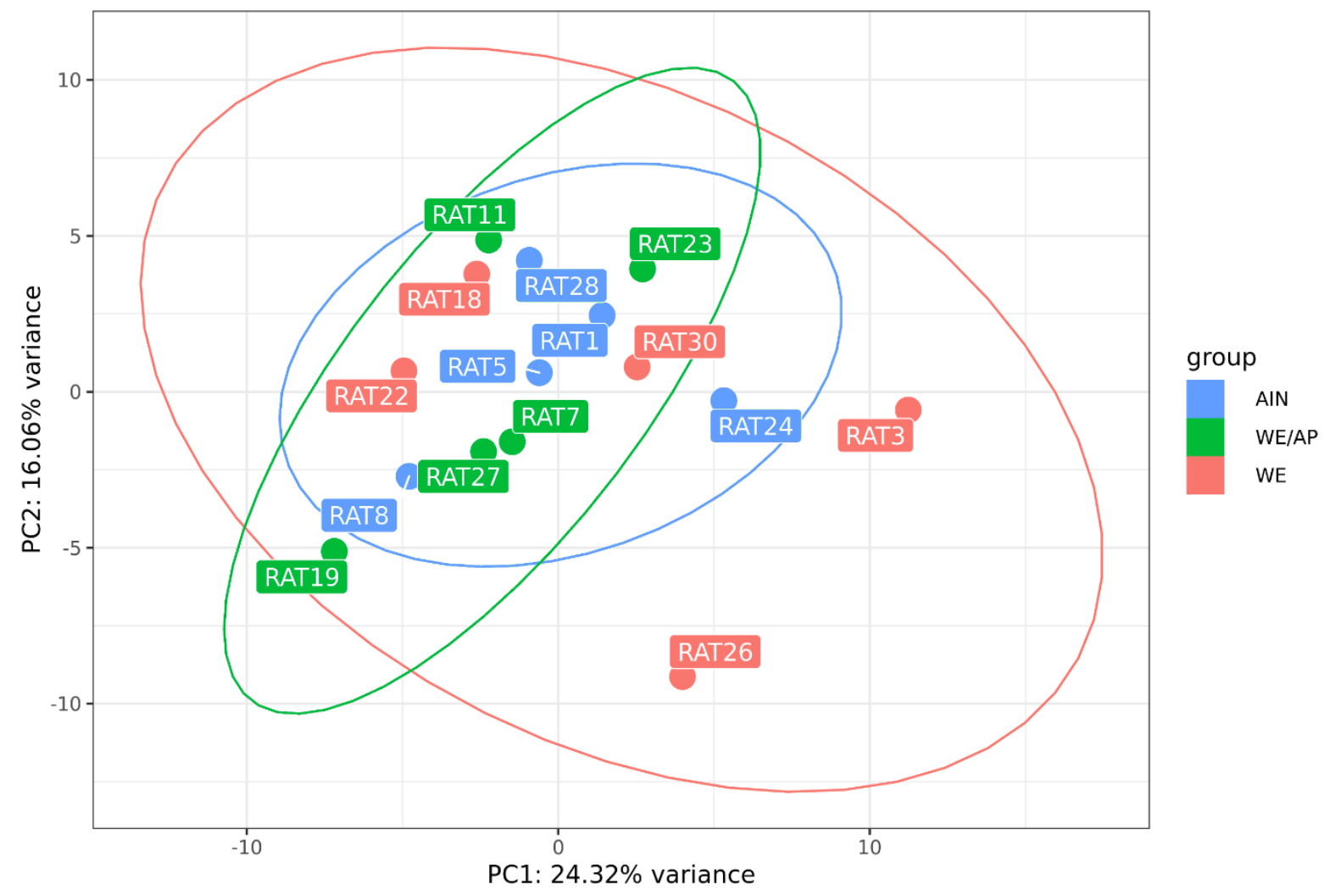


Figure 3.

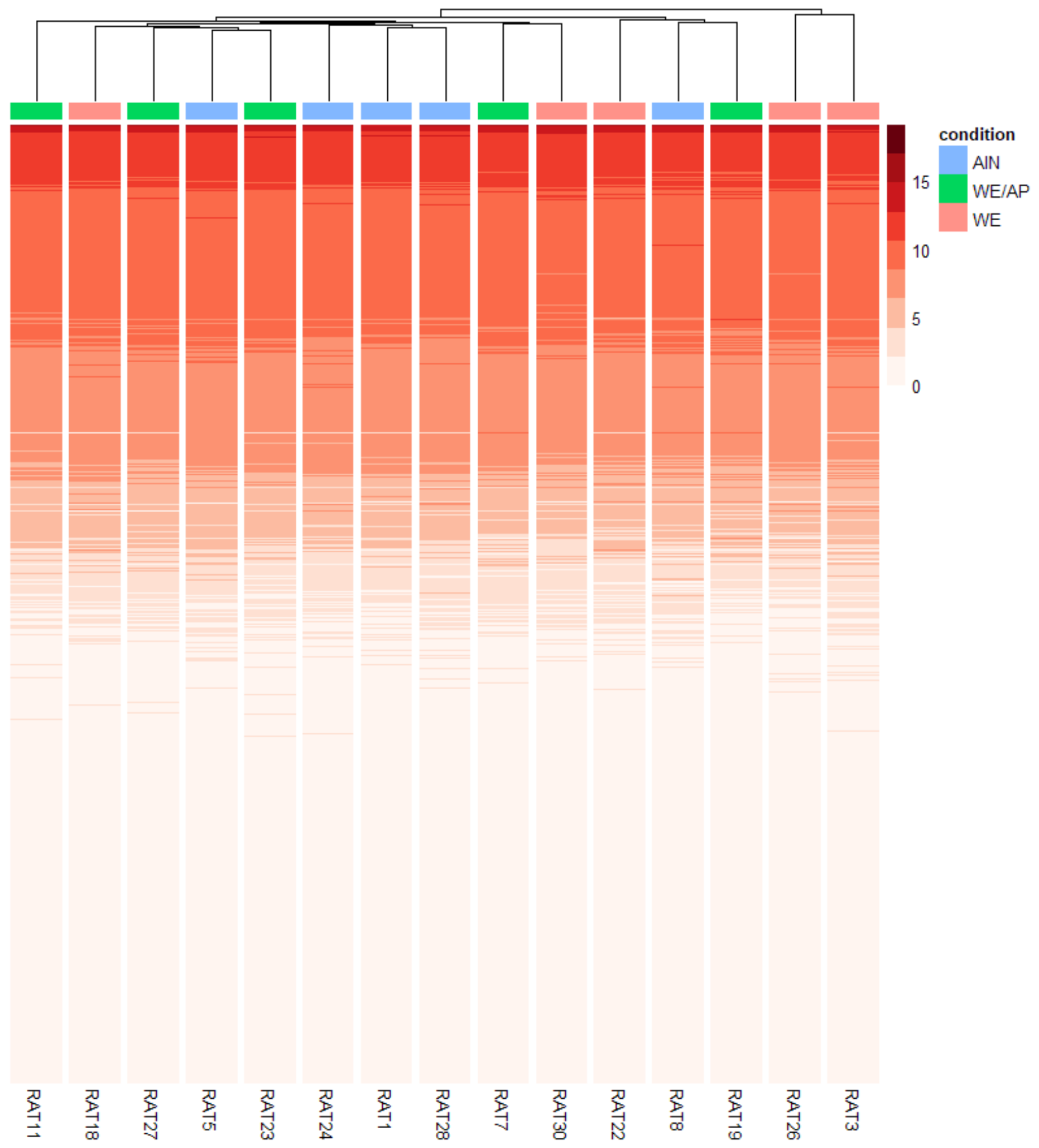


Figure 4.

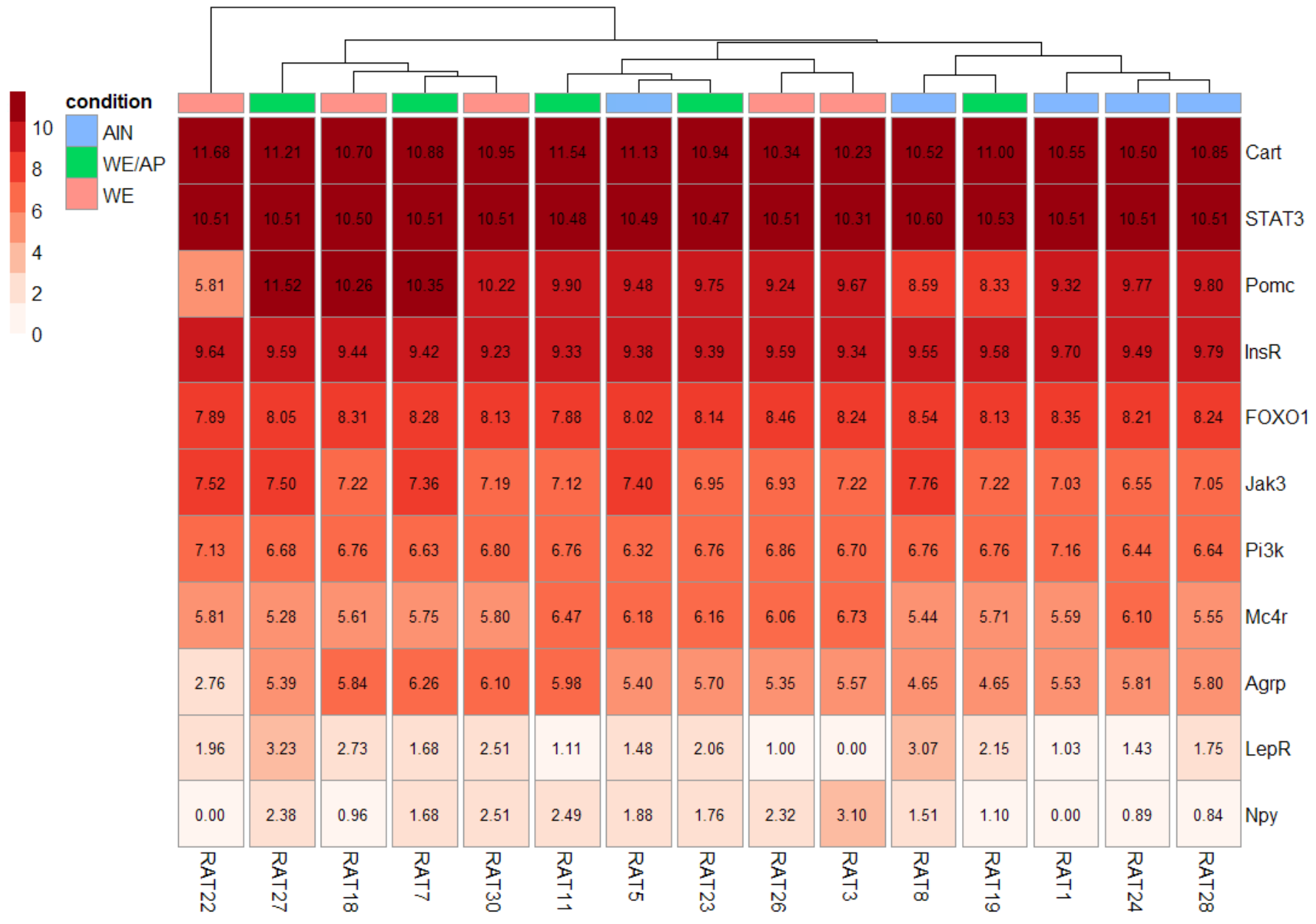


Figure 5.

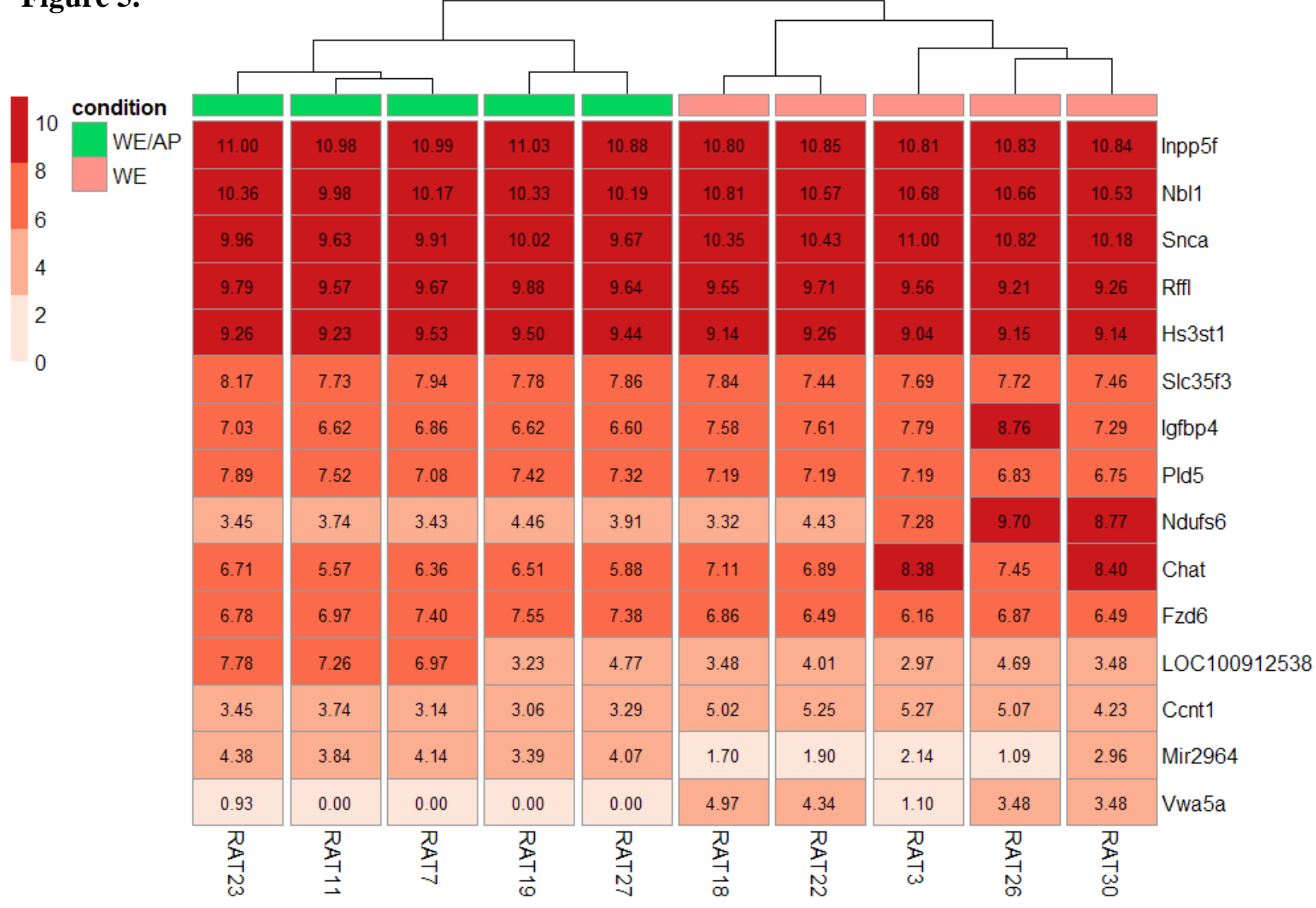




\section{Figure 6.}

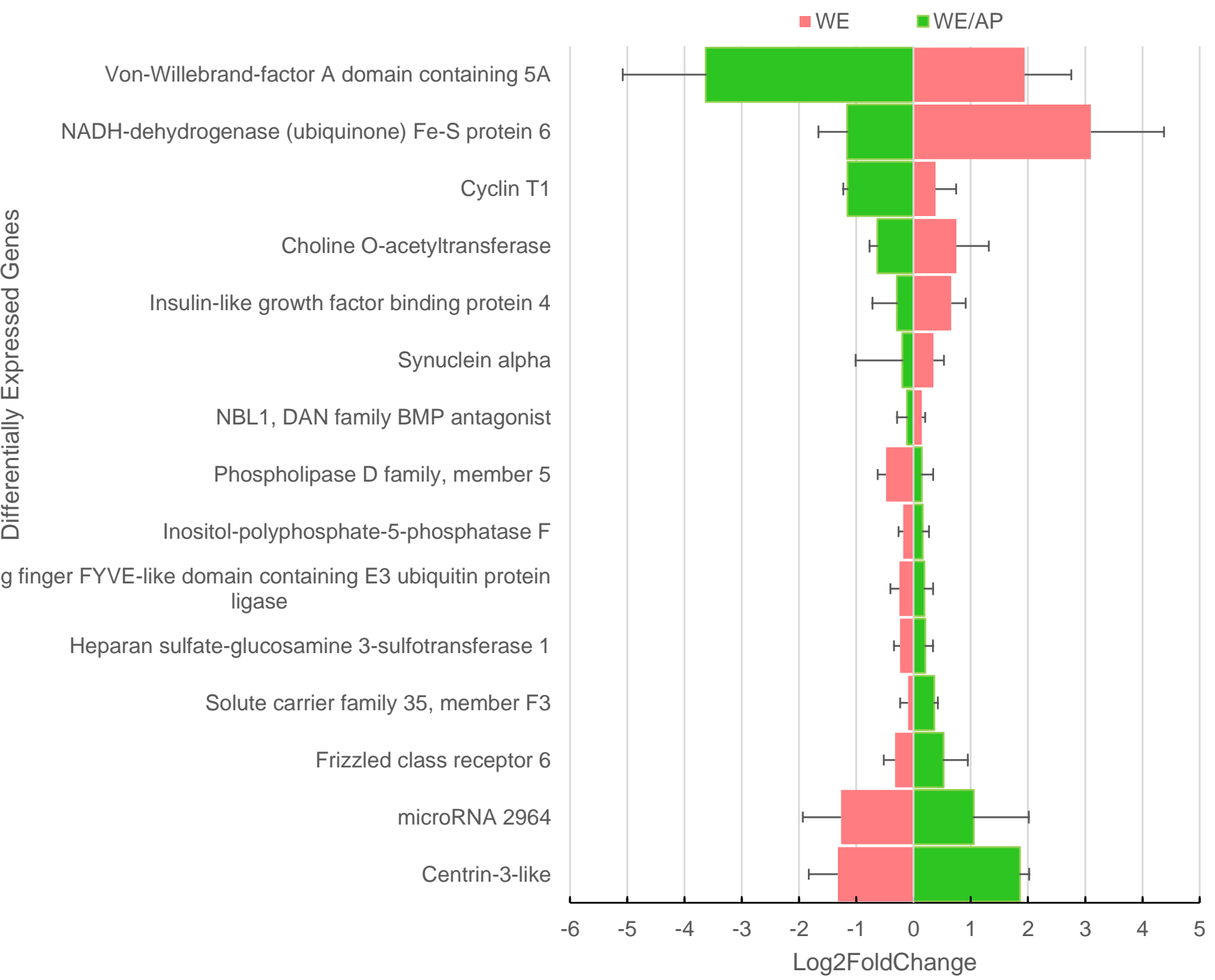




\section{Figure 7.}

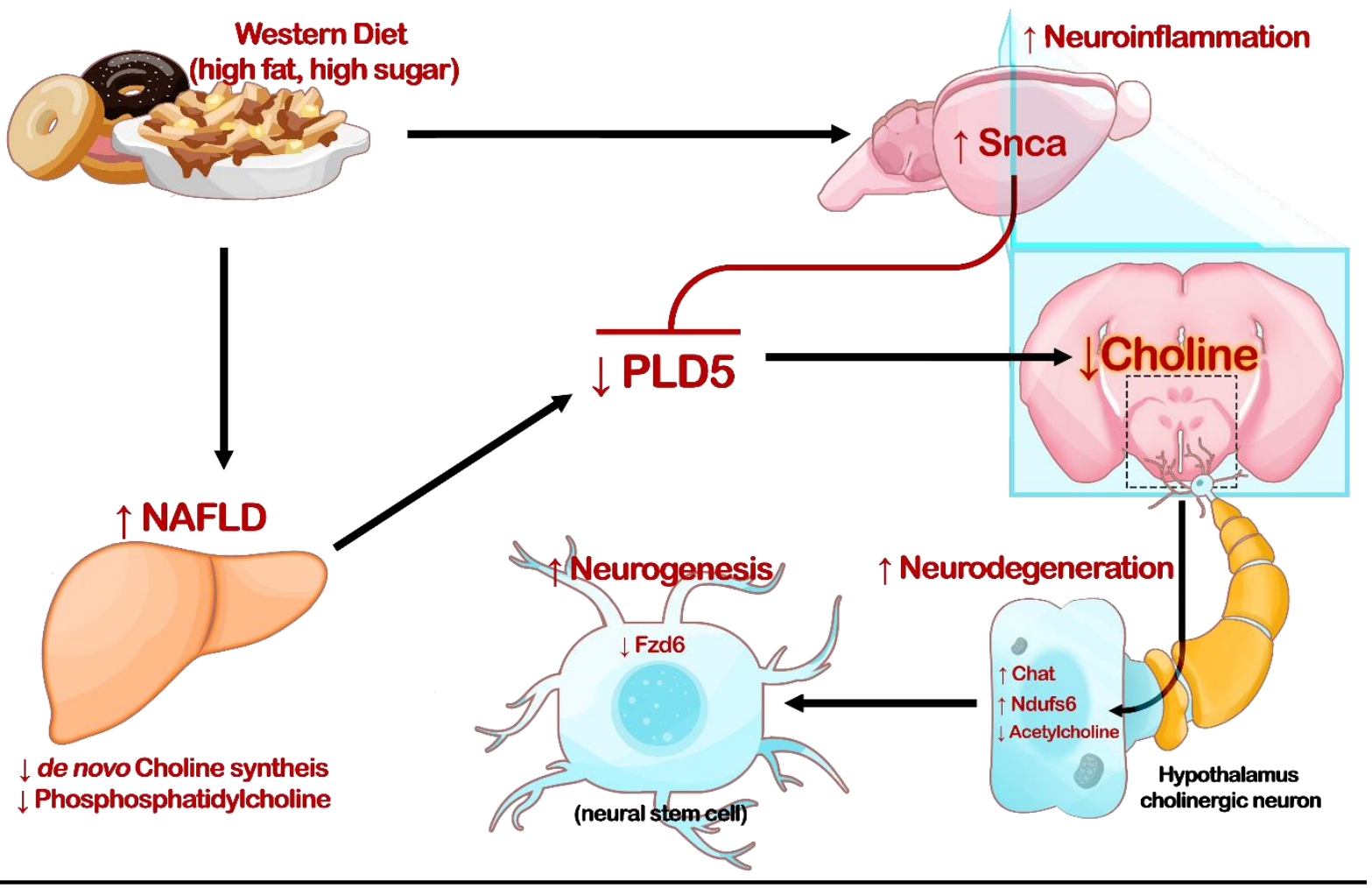

Western Diet/Apple pomace

(high fat, high sugar, solube fibers, polyphenols)

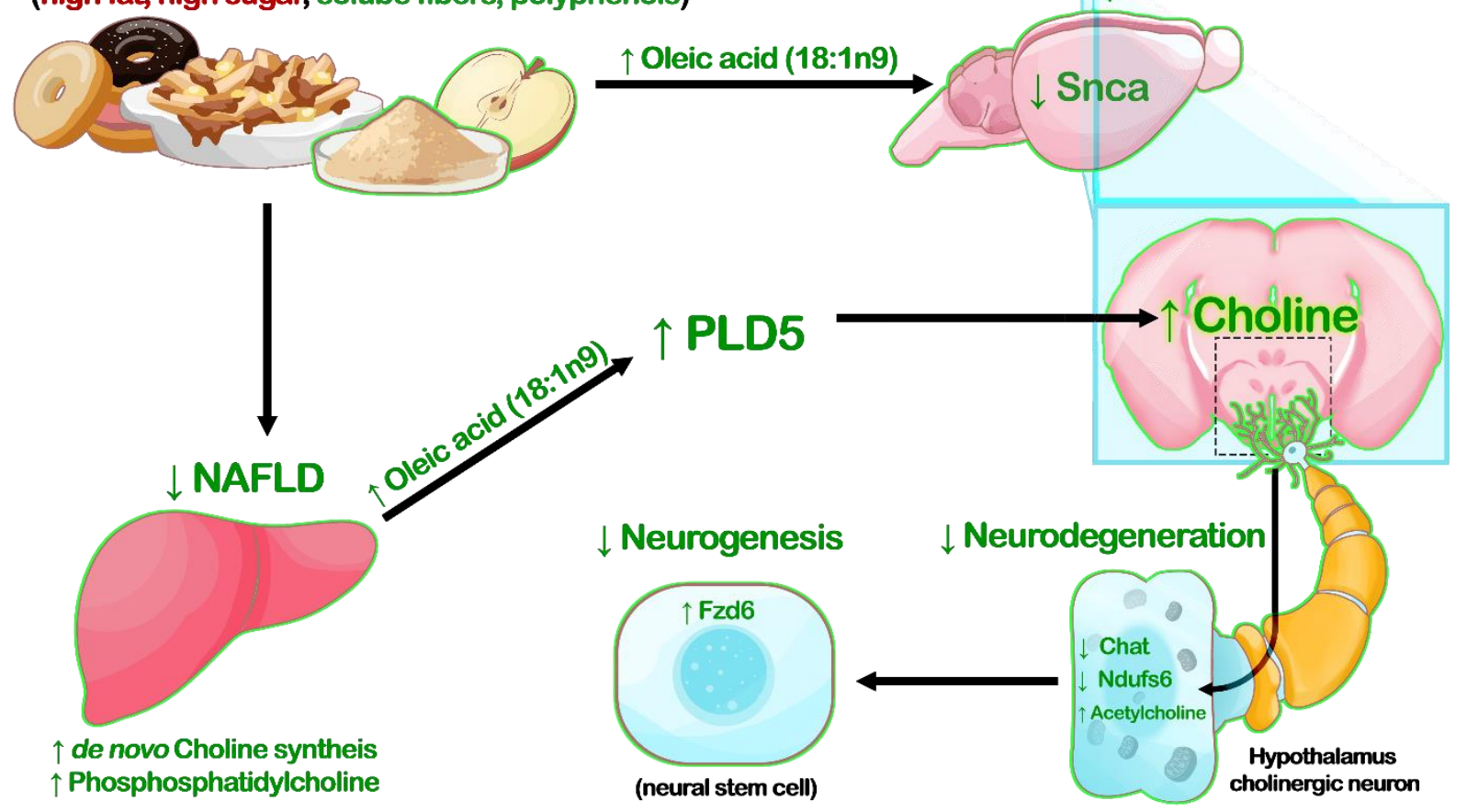




\section{Chapter 4}

\section{Future Studies}

Developing apple pomace for human consumption provides a solution for reducing the cost of apple processing waste disposal as well as health care costs for treating neurodegenerative disease. The proposed mechanism linking diet-induced NAFLD and neurodegeneration provides further insights into how diet can influence the pathogenesis of hypothalamic dysfunction and neurogenerative diseases. These insights provide a better understanding of the etiology of diet-induced neurodegenerative diseases and address this issue using sustainable dietary approaches. Studies are needed to investigate other diet-induced differentially expressed genes that were not discussed and not phenotypically implicated in hypothalamic dysfunction and neurodegeneration in this paper, which may add more information to the proposed mechanism. Future studies should also investigate the long-term effects of apple pomace consumption as a preventative therapy to attenuate the pathogenesis of neurodegeneration in animal models before conducting clinical trials. Future studies should take into consideration different developmental stages of neurodegenerative diseases when using apple pomace as a treatment. 